\title{
e-Babylab: An Open-source Browser-based Tool for Unmoderated Online Developmental Studies
}

(running head: e-Babylab)

\author{
Chang Huan Lo ${ }^{1}$ \\ Nivedita Mani ${ }^{2,3}$ \\ Natalia Kartushina ${ }^{4,5}$ \\ Julien Mayor ${ }^{4}$ \\ Jonas Hermes ${ }^{3,6}$
}

${ }^{1}$ School of Psychology, University of Nottingham Malaysia, Semenyih, Malaysia

${ }^{2}$ Psychology of Language Department, Institute of Psychology, University of Göttingen, Gosslerstr. 14, D-37073 Göttingen, Germany

${ }^{3}$ Leibniz ScienceCampus Primate Cognition, Kellnerweg 4, D-37077 Göttingen, Germany

${ }^{4}$ Department of Psychology, University of Oslo, Oslo, Norway

${ }^{5}$ Center for Multilingualism in Society across the Lifespan (Multiling), University of Oslo,

Oslo, Norway

${ }^{6}$ Department of Developmental Psychology, Institute of Psychology, University of Göttingen,

Waldweg 26, D-37073 Göttingen, Germany

Correspondence should be addressed to:

Jonas Hermes

University of Göttingen, Institute of Psychology, Department of Developmental Psychology

Waldweg 26, D- 37073 Göttingen, Germany

e-mail: jonas.hermes@psych.uni-goettingen.de 


\begin{abstract}
The COVID-19 pandemic massively changed the context and feasibility of developmental research. This new reality as well as considerations, e.g., about sample diversity and naturalistic settings for developmental research, indicate the need for solutions for online studies. In this article, we present e-Babylab, an open-source browser-based tool for unmoderated online studies specifically targeted at studying young children and babies. eBabylab offers an intuitive graphical user interface for study creation and management of studies, users, participant data, and stimulus material with no programming skills required. Various kinds of audiovisual media can be presented as stimuli and possible measures include webcam recordings, audio recordings, key presses, mouse-click/touch coordinates, and reaction times. Information pages, consent forms, and participant forms are all customizable. e-Babylab was used with a variety of measures and paradigms in 14 studies with children aged 12 months to 8 years $(n=1516)$. We briefly summarize some results of these studies to demonstrate data quality and that participants' engagement and the overall results are comparable in lab and online settings. We then present in more detail one replication of an established preferential-looking paradigm with gaze directions manually coded from the webcam captures. Finally, we discuss useful tips for using e-Babylab and present plans for upgrades.
\end{abstract}




\section{e-Babylab: An Open-source Browser-based Tool for Unmoderated Online Developmental Studies}

The year 2020 and the COVID-19 pandemic that dominated much of this year has changed the face of academia, especially in terms of increased focus on digital online formats for both teaching and research. The shutdown of laboratory testing facilities across the globe had many researchers scrambling for online tools to continue data collection in the midst of a pandemic. While this renewed drive for online testing was made further apparent in special thematic sections on platforms enabling online testing being introduced in major conferences, such sessions also served to highlight the many initiatives already in place to allow for online testing of participants. In psychological research with adult participants, the launch of Amazon's Mechanical Turk well and truly opened the door for psychological research to go online in a large scale with access to over 100,000 users from different countries, who could volunteer to participate in experiments for a fee. Furthermore, in the recent months and years, a number of tools have been set up to enable online data collection especially with regard to developmental research with children and infants (e.g., TheChildLab.com: Sheskin \& Keil, 2018; gorilla.sc: Anwyl-Irvine, Massonnié, Flitton, Kirkham, \& Evershed, 2020; jspsych.org: de Leeuw, 2015; Lookit.mit.edu: Scott \& Schulz, 2017; and discoveriesonline.org: Rhodes et al., 2020;). While there has been some debate about the issues associated with online testing and internet data collection (Hewson, Laurent, \& Vogel, 1996; Kraut et al., 2004), there are a number of associated advantages that are likely to sustain interest in online data collection in post-pandemic times.

First, collecting data online allows for greater diversity in the population sample recruited (Gosling, Vazire, Srivastava, \& John, 2004). This is especially important in the wake of recent understanding of the diversity problem in traditional psychological research, where claims about the human population at large are made from a sample of western, 
educated, industrialized, rich, and democratic societies (the WEIRD problem, Henrich, Heine, \& Norenzayan, 2010). This may be further amplified in developmental research, where research participation places additional demands on caregivers who are already pressed for time and may, therefore, self-select for caregivers from affluent backgrounds who have the time and resources to travel to a laboratory with their child to participate in an experiment for oftentimes little monetary reward. Relatedly, online data collection reduces constraints relating to the geographical location of the research institution, such as regional or even national borders, (Lourenco \& Tasimi, 2020; Sheskin et al., 2020), and allows for the same study to be run in different countries paving the way for cross-cultural collaborations (c.f. the current ManyBabies at home initiative ${ }^{1}$ ). Where needed, study material can be translated into different languages, while retaining the same study setup, allowing for direct replications across different linguistic environments (e.g., across different countries). Furthermore, since studies are administered through digital devices, such as smartphones, tablets, and laptops, studies are considerably more experimenter-independent than laboratory studies, such that experimenter effects can be kept to a minimum. With regard to promoting diversity in research output, online data collection also allows for greater equality in terms of research output allowing departments and laboratories with fewer resources access to larger sample sizes. Finally, and especially with regard to developmental research, online testing allows research to take place in the child's natural environment, bringing developmental research "to the wild". Thus, generally, and especially in times of the COVID-19 pandemic, there is a need to develop an efficient infrastructure for online testing (Sauter, Draschkow, \& Mack, 2020). What are the factors, however, that need to be considered for online testing?

First, online testing can be moderated (i.e., involving live interaction with an experimenter through video chats) or unmoderated (Sheskin et al., 2020). Whether or not the

\footnotetext{
${ }^{1}$ https://manybabies.github.io/MB-AtHome/
} 
presence of an experimenter is needed depends on the paradigm to be tested. While indispensable in certain paradigms (e.g., clinical assessments, or paradigms with adaptive procedures based on verbal answers), moderated online testing has the advantage of a more natural social interaction setting. This allows the researcher to take full control of the procedure and respond individually to each child while maintaining the advantage of reduced effort for families who do not need to physically visit the laboratory for testing. On the other hand, moderated testing paradigms nevertheless necessitates scheduling of testing times and dates and cannot be conducted flexibly at the whim of the caregiver. Furthermore, such paradigms are more experimenter-dependent, raising issues of standardization. Unmoderated online testing, on the other hand, means that the procedure is fully automated and includes no live interaction. Families can participate at their convenience without the need to arrange test dates. While lacking aspects of natural social interaction and the possibility to adapt the procedure to each child (e.g., to customize the pace of the experiment), the fully automated procedure comes with high standardization and associated advantages (e.g., replicability, geographical flexibility). In addition, multiple sessions can be run in parallel, thus obviating the need for separate one-on-one sittings and freeing up resources in terms of experimenter hours.

Another factor to be considered is the scale at which a platform is deployed. On the one hand, issues of sample diversity and replication issues clearly indicate the need for collaborative decentralized testing in multiple labs as practiced already in the ManyBabies projects (Frank et al., 2017). Such collaborative projects require large-scale platforms run in collaboration with multiple labs, such as an "online CRADLE" recently proposed by a consortium of researchers (Sheskin et al., 2020).

On the other hand, and in addition to large-scale projects, smaller-scale platforms can serve important functions for piloting and establishing new procedures and paradigms. In 
particular, reservations concerning data security are a primary issue here: parents of young children, in particular, may have concerns regarding personal and audiovisual data of their young children captured in the family's private space being stored and transferred via the web. Indeed, an experiment using webcam captures for online eye-tracking with young children reported less than one percent of the website visitors actually completing the experiment (Semmelmann, Hönekopp, \& Weigelt, 2017). A small-scale platform that uses no external services and runs solely on servers of the local university may better assuage such doubts and concerns of parents regarding data security and thus lower the thresholds for participation and increase acceptance for online testing.

There is already considerable focus on the development of tools for both moderated online testing (e.g., TheChildLab.com, Sheskin \& Keil, 2018) and unmoderated online testing (e.g., gorilla.sc, and jspsych.org, Anwyl-Irvine et al., 2020; de Leeuw, 2015); targeted at developmental research with children and infants (e.g., Lookit.mit.edu, and discoveriesonline.org, Rhodes et al., 2020; Scott \& Schulz, 2017). These platforms differ with regard to the age-groups targeted or possible to test, the programming or computational skills required for experiment creation, whether or not they are commercial products, where the servers storing personal and audiovisual data of participants are located, and what kinds of measures they can record. These solutions may all provide efficient tools for online behavioral studies.

Concerning the usability of such tools, we highlight four basic requirements, which are, from our point of view, important for unmoderated online developmental studies. First, the tool needs to offer the possibility to record webcam captures of the test sessions to allow implicit and explicit responses (e.g., gaze direction, verbal answers, pointing gestures) to be captured and data quality to be evaluated. This is particularly important in research with young children who may not be able to provide manual responses (e.g., screen touches) or 
reliable spoken or written responses. Second, the tool needs to be browser-based so that parents can easily access the studies without extensive computer know-how or having to install specific software. Third, we consider it critical that the data collected from young children is hosted on a local university server without the involvement of commercial thirdparty services to ensure that the data is securely stored in accordance with data protection guidelines in different countries and also to assuage potential caregiver reservations concerning data security. Finally, given that many researchers do not have the required skills to independently program online experiments, the tool needs to include an intuitive graphical user interface (GUI) that allows even those without programming skills to create experiments based on standard paradigms of developmental research with young children and infants.

Since, to our knowledge, no present tool is capable of meeting all the above criteria, in the last years we have developed and tested e-Babylab, a new online tool which we wish to present in this article. The tool is open source with the source code available at https://github.com/lochhh/e-Babylab. The user manual is available at https://github.com/lochhh/e-Babylab/wiki. In the following sections, we will present key aspects of e-Babylab, the procedure of online studies from the participant's point of view, and an overview of the GUI for study creation (from the experimenter's point of view). We will then describe several paradigms that have been successfully tested with the tool followed by more details of a targeted replication of an established paradigm. Details of the technical underpinnings of the tool can be found in Appendix A.

\section{e-Babylab: Key Aspects}

e-Babylab is a web application that does not require the installation of any software other than a browser. The tool offers a high degree of flexibility for creating browser-based studies and has been successfully applied to a wide range of paradigms. Its intuitive GUI 
allows users to create, host, run, and manage online studies without any programming experience. Studies are highly customizable and can be translated into any language.

A variety of audiovisual media (video, audio, and image files) can be presented as study material. Measures that can be recorded include key presses, click or touch coordinates (and response latencies of these measures), and audio or video captures via the participant's webcam and microphone. Video and audio captures allow for coding of verbal answers and for manual coding of gaze direction, facial expressions as well as gestures. Media files are preloaded in the browser to enable better synchronization between the presented material and the media captures. Video and audio captures are transferred directly to the server set up at the local research facility via a secure connection (TLS, 256-bit). Since, as described above, unmoderated testing comes with the disadvantage of a lack of control over the procedure of each individual session, these media captures can be used also for post-hoc evaluation of data quality (e.g., identification of parental intervention).

Recording of video data at families' homes involves very sensitive data. Therefore, we implemented features to prevent the recording of private material not intended for upload. Throughout the study, an exit button is permanently visible at the lower right corner with which families can terminate or pause the study at any time. Furthermore, a maximum allowed duration (or timeout) can be set both at the study level (i.e., the maximum allowed duration for the entire study) and at the trial level. If a timeout is met, recording will stop and families will be redirected to either the end page or an optional pause page that allows them to either resume or terminate the study.

\section{Study Procedure}

Figure 1 illustrates the procedure of a study created in e-Babylab. The general idea is that children will sit on their parent's lap during the study. In the case of older children, parents may leave the study session once the pre-study steps are completed and consent is 
provided. Families will need a device (e.g., laptop or tablet) with Google Chrome or Mozilla Firefox installed and an internet connection. At present, experiments programmed with eBabylab are only compatible with these web browsers for Android and desktop, which make up about $82 \%$ of the browser market share worldwide (NetMarketShare, 2021). Depending on the study and the responses to be captured during the study, a webcam and/or a microphone may be required.

Each study is accessed via a Uniform Resource Locator (URL) and begins with a page containing general information and requirements of the study and the testing procedure. This is followed by an automatic browser compatibility check. In the next steps, the consent form and participant form are provided. If the study involves audio or video recording, a microphone and/or webcam setup step is included. Otherwise, the setup step is omitted. Here, the browser first requests the participant's permission to access their microphone and/or webcam. When access is given, a 3-second test audio (or test video) is recorded to ensure that both recording and uploading work. The recorded media is played back to the participants to ensure that they can be properly heard and/or seen. This procedure can be repeated, if necessary. Upon successful completion of this step, the participant is redirected to the start page of the experimental task, where they are prompted to enter full-screen mode to begin the task.

During the first trial, we usually ask parents to explicitly state their consent for study participation captured using the webcam recording. The consent can be given in a written form, as well. Next, the experimental task is presented. As described above, throughout the task, a small exit button is shown at the lower right corner of the screen, allowing the participant to quit the study at any time. If the study is configured to allow pauses, the participant, upon clicking the exit button, will be redirected to the pause page where they are 
given the option to resume or terminate the study. The end page informs participants that they have completed the study.

\section{Features}

\section{Experiment Wizard}

At the core of e-Babylab is the Experiment Wizard, a GUI with which an experiment is created (see Figure 2). The Experiment Wizard consists of five parts: general settings, HTML templates, consent form, participant form, and crucially, the experimental task.

General Settings. In general settings, the basic information related to an experiment (e.g., name, date and time of creation) is specified. In addition, the access settings, list selection strategy, and recording mode of an experiment are configured here. Specifically, an experiment - including its participants and results - can be made accessible to: a) owner only (private), b) everyone (all users), or c) group members only (group-based access control will be detailed later). As an experiment can have multiple lists (i.e., versions), three selection strategies allow experimenters to control how the lists (or versions) are distributed across participants: a) least played, in which the list where the least number of participants have participated in is always selected; b) sequential, in which lists are selected according to the order they are added to an experiment; and c) random, in which each list has the same probability of being selected, regardless of the number of participants who have participated in a given list. By selecting a recording mode, an experiment can be configured to capture: a) key presses or clicks only, b) audio and key presses or clicks, or c) video and key presses or clicks. Note that clicks may represent mouse clicks (when a mouse is used) or touches (when a touchscreen is used). These are recorded as coordinates relative to the browser window, allowing the exact locations of clicks or touches as well as the orientation of the screen to be determined. The Experiment Wizard also provides the option to include a pause page which may be useful in especially lengthy experiments. In the event that a participant fails to 
complete an experiment within a given time, or when the exit button is clicked during an experiment, rather than ending the experiment immediately, the participant will be redirected to the pause page, thus giving the participant an opportunity to resume the experiment. Any "pause" events will be recorded in the results.

HTML Templates. HTML templates allow for the customization of the looks and text (e.g., language) of all experiment webpages, including the welcome page, the consent and participant forms, the microphone and/or webcam setup pages, the experimental task page, the pause page, the error pages, as well as the end pages. A default set of HTML templates for all experiment webpages are provided for users who do not want to further customize their experiment look (see Appendix B for a sample). Alternatively, users can modify the defaults and provide their own HTML templates as well as Cascading Style Sheets (CSS) files. Customizing these templates also allows for translating the entire experiment to another language.

Consent Form. This part of the Experiment Wizard allows users to specify consent questions. These will appear on the consent form as mandatory yes-no questions. Since experiments are conducted online and the experimenter may not be physically present to ensure that consent is obtained, e-Babylab automates this by checking that all consent questions are responded with "yes". In other words, a participant is only allowed to proceed with an experiment when full consent is obtained. Otherwise, the participant will be redirected to the "Failed to obtain consent" page, which by default provides an explanation as to why they are unable to proceed with the experiment as well as the option to return to the consent form to change their responses if the responses were provided erroneously or have been revised.

Participant Form. In the participant form, personal information can be queried using different types of form fields or questions, including text fields, radio buttons, drop-down lists, 
checkboxes, and number fields. By setting fields as "required" or "optional”, users can also control which of the form items must be answered before the form can be submitted.

Experimental Task. An experimental task comes with a four-level structure (see Figure 3). At the first level are lists. Each list may represent different versions of the experiment or different conditions of a between-subjects experiment. As each experiment has its own unique URL, an added benefit of having multiple lists instead of multiple experiments is that only a single URL needs to be sent to all participants and the tool automatically distributes participants across the different experimental conditions based on the list selection strategy defined in general settings. Optionally, a list can be temporarily "deactivated", to exclude the list from being selected and distributed to future participants; this can be particularly useful when a list has had enough participants and future participants are to be distributed to other lists.

Lists are made up of outer blocks which are presented in a sequential order, and outer blocks are made up of inner blocks which can either be presented in a sequential or random order. This increases the flexibility in experimental task design. For instance, when two visual stimuli are to be presented in succession within a single trial, this trial may be represented by an inner block consisting of two trials, each presenting a visual stimulus, in either a fixed or a random order. This flexibility in presentation of stimuli in inner blocks would not be possible without the outer-inner block structure, where we would only be able to present stimuli in either a fixed or a random order, but not both. This is desirable in many experiments where introductory trials (e.g., training, familiarization) typically precede test trials, while test trials, on the other hand, are typically randomized.

At the fourth and most crucial level are trials which, as with inner blocks, can either be presented randomly or sequentially. To allow a more granular control over trial setup, the specific responses that are accepted (e.g., clicks, left arrow key, space bar) as well as the 
maximum duration of a trial are defined on a trial-level. In addition to a visual stimulus (this can be an image or a video), an audio stimulus can also be used. Stimuli presentation can be timed by setting the visual and audio onsets (in ms). By default, these values are set to 0 so that the stimuli are presented as soon as a trial begins.

\section{Experiment Management}

Experiments are managed through the Experiment Administration interface (see Figure 4), which presents a list of experiments a user has access to. Through this interface, an experiment setup can be imported and exported. This enables the sharing of experiment setups, which in turn allows experiments to be reused and adapted (e.g., for replications) with minimal effort. The results of an experiment can be downloaded here as well.

\section{Participant Management}

Participant data is managed through Participant Data Administration in which a list of participants in all experiments a user has access to is shown (see Figure 5). By clicking on a participant, users can view the participant's data, which includes the information provided in the participant form, their screen resolution, participant number, universally unique identifier (UUID; automatically assigned to distinguish participants from different experiments having the same participant number), participation date, experiment participated in, as well as list assigned. Deleting a participant removes all of their data and results.

\section{Results Output}

Results are downloaded as a ZIP archive containing an Excel (.xlsx) file for each participant and the media recordings (in .webm format, if any). Each Excel file contains two worksheets. The first contains the participant's information provided in the participant form, consent form responses, as well as aspect ratio and resolution of their screen. The second contains information for each trial, including setup information (e.g., stimuli presented, 
maximum duration allowed), the reaction times, responses given (e.g., keys pressed, mouse click coordinates), and the file names of associated media recordings.

\section{File Management}

The tool also features a file browser which allows users to create folders, upload, and manage their own study material, such as audio and visual stimuli, custom HTML templates, and CSS files (see Figure 6). The supported file extensions for each of the allowed file types can be found in Table 1.

\section{Authentication and Authorization}

Access to e-Babylab and its data is secured by authentication and authorization. Essentially, authentication verifies the identity of a user and authorization determines the operations an authenticated user can perform on a system (i.e., access rights). Two types of user accounts are offered: normal user and administrator. By default, an administrator has all permissions needed to perform particular functions within e-Babylab (e.g., adding a user, changing an experiment, assigning permissions) without explicitly assigning them. A normal user, on the other hand, does not have any permissions, but instead requires permissions to be assigned by another user who has the permission to do so (e.g., an administrator).

\section{Group-Based Access Control}

An experiment, including its participant data and results, can be made accessible to other users through groups. For instance, a group can be created for a particular research group or laboratory and an experiment can be shared among all users belonging to this group. As permissions can be assigned on a group-level, groups can also be used to more efficiently manage access rights by assigning users to groups. In other words, a user need not be directly assigned permissions, but rather acquire them through their assigned group(s). 


\section{Overview of Paradigms Successfully Run on e-Babylab}

An overview of the tasks run on e-Babylab related to projects launched by the BabyLing lab at the University of Oslo (NK and JM) is presented in Table 2. As can be seen in the table, at the time of writing, nine studies were implemented on e-Babylab, resulting in a total of 1103 children (from 18 months to 6 years) being tested. In addition, three studies using looking time data and two studies using behavioral data or verbal responses were run at the University of Göttingen (NM \& JH), resulting in a total of 413 children (from 12 months to 8 years). We only provide data from one of these looking time studies in detail in the interest of brevity. At the University of Nottingham Malaysia, e-Babylab was also used to simultaneously collect visual and haptic responses in examining 32 30-month-olds' word learning from tablets (Study 2; Ackermann, Lo, Mani, \& Mayor, 2020). ${ }^{2}$ Below, to illustrate children's engagement and performance in the tasks, we present a detailed analysis of some data collected via e-Babylab and complement it with some tips that future users may find useful.

\section{N-Alternative Forced-Choice Identification Tasks}

\section{Engagement}

To illustrate children's engagement in the tasks implemented on e-Babylab, we analyzed touch responses in 49 Norwegian 18-20-month-old toddlers performing a 2alternative forced-choice word recognition task, a study referred to as the Toddler-based CDI in Table 2 (Lo, Rosslund, Chai, Mayor, \& Kartushina, in press). On each trial, two familiar objects (e.g., a dog and a plane) were presented on the screen, followed by a prompt instructing toddlers to select one of them (“Can you touch the dog?"). There were 48 trials in total.

\footnotetext{
${ }^{2}$ An adapted version of e-Babylab was used in this study to dynamically generate trials based on toddlers' responses.
} 
The number of trials in which a touch response was produced, regardless of the accuracy of the response, was used as a measure of toddlers' motivation to produce a response during the word recognition task. On average, toddlers attempted to provide an answer on 42 out of 48 available trials. The number of touch responses increased with age $(r=.31, p=.03)$, with 40 and 46 touch responses produced, on an average, by 18-month-old and 20-month-old toddlers, respectively. Trials containing difficult words - those that were known by less than $20 \%$ of toddlers as reported by parents in the Norwegian version of the CDI (Simonsen, Kristoffersen, Bleses, Wehberg, \& Jørgensen, 2014) - elicited less touch responses, i.e., 87\% of trials $(S D=18)$, than trials containing easy words (reportedly known by more than $80 \%$ of 20-month-old toddlers), i.e., $91 \%$ of trials $(S D=13), t(48)=-2.312, p=.0248$. Anecdotally, around $5 \%$ of parents reported having run the task several times, as their child liked 'to play' and wanted to do the task again. ${ }^{3}$ These results suggest that toddlers were engaged in the task and that their responses were non-random.

\section{Home Versus Lab Setting}

In the same study (Toddler-based CDI), and due to the Covid-19 outbreak, 28 out of 49 toddlers performed the task at their homes, on their parents' touchscreen devices, thus allowing for a comparison of toddlers' engagement and accuracy between lab and home/online test settings. A comparison of the number of attempted trials did not reveal significant differences between children who were tested online $(M=44, S D=6.3)$ and in the lab $(M=41, S D=7), t(40.6)=-1.78 ; p=.083$. Likewise, a comparison of the number of accurately identified items between the two settings revealed no significant differences between the two groups of toddlers (online: $M=38, S D=7.26$ and lab: $M=34, S D=8.72$ ), $t(38.5)=-1.78 ; \mathrm{p}=.082$. In sum, these results suggest there was no evidence for differences

\footnotetext{
${ }^{3}$ Note that only the first attempt was used for the analyses.
} 
in accuracy and the degree of motivation to complete the task across toddlers tested in the lab and at home.

\section{Processing and Reaction Times}

To examine reaction times as a function of age and familiarity with the task, we examined touch latencies for accurate answers in 106 Norwegian 2-6-year-old children $(M=$ 3.7 years, $S D=1.14$ years). These children completed a 4 -alternative forced-choice word recognition task in their kindergarten. Here, they were presented with four familiar objects (apple, dog, car and ball, see Figure 7), used as control trials as part of a larger study. On each trial, children saw four items and were instructed, by an audio prompt, to touch the named target. The timeout was set to 20 s. Children performed the task on a Samsung Galaxy Tab S4, twice in the academic year, in September and June, with a 10-month interval between the two times. Accidental touches (defined as taking place $1.5 \mathrm{~s}$ and less after name onset) were removed from the analyses, in line with previous research (Ackermann et al., 2020).

Children's reaction times ranged from 1.74 to $9.92 \mathrm{~s}(M=4.15 \mathrm{~s}, S D=1.64 \mathrm{~s})$.

To assess the dynamics of children's response latencies across time and age, we performed a linear mixed-effect regression model, using lmer function in the lme 4 package (Bates, Mächler, Bolker, \& Walker, 2015), with the fixed factors Time (Time 1 and 2), Age (in months), and Trial; the random factors included Child, adjusted for the effects of Time, and Word ${ }^{4}$. The dependent variable was log-transformed to meet the assumptions of a normal distribution. The results are summarized in Table 3; the significant effects of Age, Time, and Trial indicate, respectively, that (1) older children answered faster than younger children, (2) reaction times were faster at Time 2, as compared to Time 1 (see Figure 8) and (3) later trials yield faster responses.

\footnotetext{
${ }^{4}$ The model including a Time|Stimulus random slope did not converge.
} 
Average reaction times per age and at each testing time are summarized in Table 4. These results indicate that children answered faster at Time 2, as compared to Time 1, even when age was controlled for. Thus, prior experience with the task, and the touchscreen paradigm, had long-lasting beneficial effects on reaction times when performing the task for the second time. The effect of prior experience with the task varied across ages and ranged between 0.18 and $0.68 \mathrm{~s}$. A very recent study has similarly reported that experience with paradigms affects infants' behavior in the task: e.g., more experience with the head-turn preference paradigm (more lab visits) leads to smaller familiarity preference, i.e., smaller effect sizes, suggesting that prior experience with the task cumulates and modulates the learning outcome (Santolin, Garcia-Castro, Zettersten, Sebastian-Galles, \& Saffran, 2021).

\section{Looking Time Tasks}

There are age-related effects on children's provision of reliable touchscreen and spoken responses, with young children providing fewer and slower touchscreen responses (leave alone issues related to mouse or keyboard responses) and also being too shy to produce spoken responses with either the experimenter or the parent (see below). Recognizing such issues even in laboratory-based tests (Golinkoff, Ma, Song, \& Hirsh-Pasek, 2013 for issues with tasks requiring explicit responses from young children), most paradigms with young infants have relied on collecting data of infants' eye movements as an implicit measure of children's processing of audio and visual information presented in the study. A seismic change in this regard was brought about with the introduction of the intermodal preferential looking paradigm, where children were presented with audiovisual input and their eye movements across a screen were analyzed as an index of their recognition of their relationship between the auditory and visual input (Golinkoff, Hirsh-Pasek, Cauley, \& Gordon, 1987, following work by ; Spelke, 1979). Thus, we consider it critical that online testing tools also 
allow for examination of children's implicit processing of audiovisual input. e-Babylab allows for this by requesting participants' permission to access their webcam and/or microphone and capturing video or audio of participants on a trial-by-trial basis during the study.

We capitalized on this possibility for an online replication of a standard looking time task examining whether young children can use thematic information provided in the input to anticipate upcoming linguistic input and use this to fixate a target image prior to it being explicitly named (Mani \& Huettig, 2012). Thus, here, participants are presented with images of two familiar name-known objects, e.g., a cake and a bird, and hear the sentence "The boy eats the big cake". The verb eat thematically constrains how the sentence will be continued, with only edible nouns constituting permissible continuations of the input thus far. Thus, cake is a more suitable continuation of the sentence relative to bird. If children are sensitive to such thematic constraints and can use them to anticipate upcoming linguistic input, we expect them to fixate the target object cake soon after the verb is presented but critically, prior to the target label being presented. Laboratory-based tasks show robust prediction of upcoming linguistic input (as described above) in two-year-olds (Bobb, Huettig, \& Mani, 2016; Borovsky, Elman, \& Fernald, 2012; Mani, Daum, \& Huettig, 2016; Mani \& Huettig, 2012). This paradigm requires us to be able to capture the swift pace of linguistic processing since participants have a few hundred ms between the offset of the verb and the onset of the target to demonstrate a prediction response. It provides, therefore, a good test of e-Babylab's suitability to capture the time-course of linguistic processing.

\section{Participants}

Participants were children whose caregivers had previously provided us with their details and agreed to be contacted by us for participation in our laboratory studies. We invited families to participate after they had completed another study in our laboratory. Caregivers' written consent was obtained. We tested 26 participants aged $2-8$ years $(M=55$ months, $S D=$ 
16 months, range: 28 - 101 months). Of these, we obtained data form 25 participants - data from one participant was excluded because the videos were longer than expected suggesting technical issues with regard to the timing of stimuli presentation. Participants (in this study, see below) were tested at our laboratory testing facility as the focus of this study was to examine the usability of the looking time data collected.

\section{Stimuli}

The stimuli presented to children in the study were identical to that used in Mani \& Huettig (2012). Thus, we uploaded the auditory files and the visual images from that study to e-Babylab.

\section{Procedure}

Children and their caregivers were led to a room and seated in front of a laptop where the experiment was presented. Children were sitting on their caregivers' lap; some of the older children were sitting alone. The experimenter explained the purpose of the study (i.e., to test the usability of a new tool) and remained in the background available for questions while the caregivers and participants completed the steps of the online experiment (see above). In the participant form, we asked caregivers to provide their child's name and age in years. After the consent form, the webcam setup, and the study information were presented, caregivers again expressed their consent to participation by reading out a text while being recorded with their child on their lap via webcam (this was programmed as the first trial in e-Babylab). Before the task began, caregivers were informed that pictures would be shown, sentences would be heard, and that children were only required to look at the stimuli presented.

The set-up of the task was identical to Mani \& Huettig (2012). ${ }^{5}$ Throughout the task, the participant's response to the presented stimuli was recorded via a webcam and a

\footnotetext{
${ }^{5}$ For brevity, we include only the details needed for interpretation of the results presented here.
} 
microphone. Each participant was presented with 12 trials, six of which were experimental trials, where the thematic constraints of the verb were more consistent with one of the images on the screen (e.g., eat-cake), and six of which were control trials (e.g., see-cake), where the thematic constraints of the verb were equally consistent with both images onscreen. The images were presented in silence before the sentence began such that the onset of the verb was $3000 \mathrm{~ms}$ into the trial. The sentence (e.g., "The boy eats the big cake") continued with the onset of the critical target label at different points in the trial. Whether a boy or a girl was mentioned and which stimuli were presented as experimental or control trials were counterbalanced between subjects. Order of the trials was randomized. ${ }^{6}$

\section{Data Preprocessing and Analyses}

The videos for each individual trial for each participant were coded by two blind coders who were blind to the trial conditions. Videos were coded in ELAN ${ }^{7}$ (Lausberg \& Sloetjes, 2009). Since we were interested in the usability of video data obtained and the extent to which we could reliably code where participants were looking at any point in the trial, the two blind coders coded all trials in the study. Coders were asked to code whether children were looking to the left or right side of the screen or away from the screen. Looks were then recoded in $\mathrm{R}$ as to whether participants were looking at the target (the later labelled object) or the distractor. Thus, coders indicated the onset and offset of a look to the left and right of the screen (in a trial), from which we calculated the proportion of target looking (proportion of looks to the target relative to the looks to the target and distractor) for each $20 \mathrm{~ms}$ bin of the trial. Cohen's kappa for inter-rater reliability suggested almost perfect agreement between raters, $K=.993, p<.001$, suggesting that the quality of video data collected was adequate to allow reliable coding of whether participants were looking to the left or right of the screen.

\footnotetext{
${ }^{6}$ This study was conducted on a previous version of e-Babylab.

${ }^{7}$ ELAN (Version 5.9) [Computer software]. (2020). Nijmegen: Max Planck Institute for Psycholinguistics, The Language Archive. Retrieved from https://archive.mpi.nl/tla/elan
} 
We included three main time windows in the analyses: the pre-verb window, the verb window, and the noun window. The pre-verb window considered all fixations in the $2000 \mathrm{~ms}$ prior to the onset of the verb (i.e., 1000 to $3000 \mathrm{~ms}$ into the trial), the verb window considered all fixations from the onset of the verb (e.g., eat) till the onset of the noun (e.g., cake), while the noun window considered all fixations from the onset of the noun till $2000 \mathrm{~ms}$ after the onset of the noun. As in the original study, we ran a $2 \times 3$ ANOVA with Condition (semantically constraining, semantically neutral) and Window (pre-verb, verb, and noun window) as within-subjects factors. We predicted that children would look more to the target in the verb window (relative to the pre-verb window) when the verb was consistent with one of the two images on screen (semantically constraining trials, e.g., eat-cake), relative to where the verb was consistent with both images on screen (semantically neutral trials, e.g., seecake). In contrast, we predicted no difference between the two conditions in the pre-verb window and the noun window.

We coded video data for 297 of 312 trials presented to children (26 participants each presented with 12 trials), with a mean of 11.42 trials per participant ( $S D=1.73$, Range: $4-12$ ). The video data of two participants were incomplete (4 and 8 trials missing, respectively) because of technical errors during the video transfer. Three additional trials were not included in the analysis due to coder error or technical error during coding. And the data of one child (12 trials) were excluded due to technical error (see above), resulting in 285 trials for analysis. During the critical time windows, participants spent $89 \%$ of the time looking at either the target $(56 \%)$ or the distractor $(35 \%)$ and only $8 \%$ of the time not looking either to the left or right of the screen, suggesting that they were paying attention to the information being presented. When considering the data across the entire trial, they spent $27 \%$ looking at the distractor, $41 \%$ looking at the target, and 33\% looking neither at the target nor distractor.

\section{Results and Discussion}


We replicated the findings of the original study (Mani \& Huettig, 2012), with a main effect of Condition (semantically constraining, semantically neutral; $F(1,24)=9.96, p=.004$ ) and Window (pre-, verb-, noun-windows; $F(3,72)=25.79, p<.001)$ as well as an interaction between Condition and Window, $F(3,72)=5.39, p=.002$. Follow-up t-tests found no difference between conditions in the pre-naming, $t(24)=-.55, p=.585$, or the noun window, $t(24)=-1.34, p=.192$, but a significant difference between conditions in the verb window, $t(24)=-6.32, p<.001$. There was also a significant difference between the semantically constraining and semantically neutral trials in terms of the difference between the pre-naming and verb windows, $F(1,24)=8.39, p=.007$. Figure 9 shows the time course of fixations to the target across the critical time window. As Figure 9 suggests, the fixations diverge shortly after the onset of the verb and before the earliest onset of the disambiguating noun. More specifically, children fixated the thematically related target only in semantically constraining trials but not in semantically neutral trials, and this remained distinct for the first $1000 \mathrm{~ms}$ of the beginning of the noun window.

Our replication of the results of the original study highlights the viability of e-Babylab for such fine-grained studies examining the dynamics of infants' eye movements across the screen over time. The timing of the effect in Figure 9, a few hundred ms after the onset of the verb particularly highlights the efficacy of the tool even for studies examining rapidly changing stimuli like speech processing. In what follows, we will not discuss the findings in general, but will focus instead on the efficacy of the tool for such online looking time tasks (as well as for verbal and behavioral data coded from video recordings).

One of the first caveats of the study mentioned above is that participants were tested online in a laboratory with a stable internet connection. It is, therefore, important to know to what extent similar data quality is to be expected when children are tested in the comfort of their own homes. With this in mind, we refer to two additional online looking time studies 
that we have run using the same tool. In one study, we presented infants with multiple objects from two categories and then examined infants' categorization of the objects to the extent that they distinguished between a novel object of one of the familiarized categories and an unfamiliar object from a different category (Bothe, Trouillet, Elsner, \& Mani, in prep). Each child (either 1- or 2-years of age) was presented with 1010 -second long training trials and 2 10 -second long test trials (and three attention-getters that we do not report on further). Thus far, a total of 176 children have been presented with 1760 trials altogether, from which we obtained video data for 1454 trials (82\%) and were able to reliably code data for 1198 trials ( $82 \%$ of the trials we obtained video data for). Inability to code was typically due to poor lighting or the child not being positioned properly (e.g., not showing both eyes clearly). This led to a dropout (of individual participants) of around $28 \%$ at both age-groups (28.08\% at 12 months and $28.39 \%$ at 24-months). In another study, we presented 52 children with 22 trials (that needed to be coded) and an additional 12 trials for which we did not need to code the data (34 trials altogether). We obtained video data for 1584 out of 1768 trials (89\%). We excluded 12 children from further coding (23\% dropout) because they did not provide us data for critical trials in the study, leaving us with 40 children who provided us with 880 trials that needed to be coded. Of these, we could reliably code 851 trials (96\%). Altogether, this suggests a relatively high dropout rate (more on this below) of around $25 \%$ of children whom, across the looking time studies, we were unable to include in further analyses.

Across two additional yet unpublished studies run on e-Babylab with children aged 2 to 6 years in which webcam captures were coded for behavioral data and verbal responses, video recordings were incomplete for 54 out of 149 participants (36\%). Out of the 7752 trials presented across these two studies, we obtained video data for 6868 trials (89\%). Missing video recordings resulted in a 3\% dropout rate (5 participants) and recordings of all participants except for one (whose recordings were mute) could be coded. 
Notwithstanding high rates of video loss and dropouts, the data obtained from eBabylab can be reliably coded as between $82 \%$ to $96 \%$ of trials were included in the analyses, ${ }^{8}$ suggesting acceptable efficacy of e-Babylab in children's natural environments.

\section{Lessons or Tips: What We Have Learnt}

\section{Volume Adjustment}

For designs containing audio stimuli, it is important to let participants adjust the volume before the test. For this purpose, we used - and recommend future users to include - at the beginning of a task, a trial with an audio playing continuously and with no timeout, instructing participants to adjust the volume to a pleasant (for their child) level.

\section{Display Mode}

For tasks performed remotely and involving the use of handheld devices (e.g., tablets, smartphones), participants (or caregivers) need to be instructed, at the beginning of the study, to hold their device either in portrait or landscape display mode (depending on the design of the task) and to not re-orient the device during the task.

\section{Browser}

Before participants are directed to the experiment URL, they need to be instructed to open it with a compatible browser (i.e., Chrome or Mozilla Firefox) and device (e.g., no iPads - see Media Recording in Appendix A).

\section{Technical Considerations}

We recommend the use of Handbrake (https://handbrake.fr/) for optimising video stimuli for web pages and converting these to formats that keep the file sizes small without compromising on quality (e.g., .mp4). A stable internet connection is a prerequisite for

\footnotetext{
${ }^{8}$ We do not provide details of the results of these studies due to the data being privileged to written manuscripts of these studies
} 
participating in an e-Babylab study. Internet data usage varies depending on the size of the study. For instance, a study that uses video stimuli and records videos of participants will require much more data than one that presents images and records only screen touches.

\section{Data Processing}

To process raw data saved in .xlsx files, researchers can use R, MATLAB, or built-in functions in Microsoft Excel. For our research projects, we created a couple of "template" R and MATLAB scripts that computed accuracies from mouse-click/touch coordinates or calculated where infants were looking in each trial. Some of these scripts are available on the OSF pages of the projects (see Table 1). For the majority of the projects, we analyzed accuracy and looking time data only. However, note, that even when not analyzing response times explicitly, these can be useful when processing accuracy data, for instance, to exclude accidental touches (when motor response is faster than $1.5 \mathrm{~s}$ ) or extremely long latencies.

\section{Audio Recordings}

Some of our projects involved the recording of participants producing a verbal response. Although, overall, children were engaged in an object naming task, we observed that very few responses were obtained from 2- and 3-year-olds, who were typically too shy to provide verbal responses to an experimenter (or to their parent). To examine the quality of the audio recording obtained via e-Babylab, we computed an average speech-to-noise ratio $(\mathrm{SNR})^{9}$, on a sample of eight randomly selected recordings from an emotion word production task (see Table 1). The results revealed an average $\mathrm{SNR}=54 \mathrm{~dB}(\mathrm{SD}=16.4)$; note that the SNR of $50 \mathrm{~dB}$ and higher is considered to be a very good quality recording.

\section{Video Recordings}

\footnotetext{
${ }^{9}$ The following formula was used: SNR $=20 \log$ (Power_signal/Power_noise), where log is 10-based.
} 
Some of our projects involved the recording of participants' eye movements. We noted that we suffered some data loss due to coders not being able to see the child's eyes or assess where the child was looking. Here, we suggest it might be good to inform the parent beforehand that it is important that we need to be able to clearly see the child's eyes and to ask them to ascertain this during the video check at the beginning of the study. Instructions sent to parents could also include specific details with regard to lighting in the room where the study takes place.

Additionally, probably due to participants' poor internet connections, we experienced an issue with video recordings not being uploaded to the server. We found this issue to occur more frequently when single trials were rather long. Therefore, we recommended keeping trials short (ideally less than $30 \mathrm{~s}$ ) or, if possible, splitting longer trials into a sequence several shorter trials within an outer block.

\section{Trial Timing}

We observed that participant videos obtained during the studies involving stimuli with large file sizes (e.g., video) were typically off by up to a few hundred ms. This was largely due to data bandwidth issues which led to delays in the onset of stimulus presentation. While this issue can be worked around by coding when stimulus presentation starts and ends in each trial - which is clearly visible due to the change in the lighting on the face of the child - we advocate for a conservative use of data resources, and use stimuli with modest file sizes.

\section{Planned Features}

A series of features are being evaluated or planned, in order to make e-Babylab even more user-friendly and efficient. At the time of writing, they are not part of the release: (1) allow for the names of output files (e.g., excel, audio) to be "personalised", so as to contain participants' IDs and the date of test, (2) record screen orientation for each trial, as some parents and/or children may turn the handheld device around during the task, thus making the 
identification of the selected object from the screen location more difficult, (3) include the opportunity to inspect individual participants' answers online, without the need to download data for all participants, (4) reduce the necessary bandwidth when participants have reduced internet speed by potentially delaying participants video upload until the end of the study, thereby reducing data loss and potential lags between video stimulus presentation and video recording, (5) offer creation of areas of interest so that mouse-clicks/screen touches can be automatically coded without the need for external scripts, (6) integrate an HTML editor for easier modification of HTML templates, (7) allow greater degrees of freedom with regard to counterbalancing and randomisation of trials, (8) allow for different kinds of responses (e.g., video/audio recordings) to be collected in different trials, and finally (9) integrate adaptive structures (e.g., if participant responds with $\mathrm{Y}$, go to trial n). We are currently working on implementation of these changes and beta-testing once these changes have been implemented. In addition, we are also looking into the possibility of automatic real-time gaze detection so that gaze data can be directly obtained, thus obviating the need for manual gaze coding and for transferring video data to the server. Finally, given recent innovations with regard to digitalised communicative developmental inventories (e.g., Chai, Lo, \& Mayor, 2020; Mayor \& Mani, 2019), we also plan to expand e-Babylab to incorporate such tools so that we can hopefully present a one-stop tool to developmental researchers.

In conclusion, we present here a highly flexible tool that allows researchers to create and conduct online studies using a wide range of measures. We also demonstrate the efficacy of the tool with regard to the data quality. Importantly, we highlight a number of use cases for e-Babylab particularly in developmental research. With regard to the aforementioned criteria for an interface suitable for developmental research, e-Babylab allows the possibility of recording webcam videos of the test sessions and brings looking time tasks to the child's home. In addition, e-Babylab is browser-based and thus does not require participants to install 
additional software or to possess extensive computer know-how to be able to take part in studies. Further, the data collected is stored on local university servers (of the respective groups using e-Babylab). We believe this latter point is particularly important in developmental research, since parents of young children may have reservations concerning data security. Finally, given that many researchers do not have the required skills to independently program online studies, e-Babylab provides a highly intuitive graphical user interface that allows even those without programming skills to conduct online studies. 


\section{Open Practices Statement}

All code to set up e-Babylab as well as a user manual are available on GitHub (https://github.com/lochhh/e-Babylab; https://github.com/lochhh/e-Babylab/wiki).

The data of the study presented in the section Looking Time Tasks was not preregistered and will be made available in a data repository upon publication of this article, the RMarkdown file is available at https://osf.io/gqep8/. Most of the data of the additional experiments mentioned in this article were preregistered. We refer to the (upcoming) preprints and articles for more details (see Table 1).

\section{Acknowledgements}

Development of e-Babylab was funded by a Leibniz Science Campus Primate Cognition seed fund grant awarded to JH \& NM and by the FRGS grant awarded to JM: FRGS-NFHY0004. NK's work was partly supported by the Research Council of Norway through its Centres of Excellence funding scheme, project number 223265. Thank you very much to Monja Krücke, Marieke Albrecht, Ronja Limburg, Natalie Bleijlevens, Evin Yildirim, Audun Rosslund, and Ane Theimann for their help with running studies, coding, or sharing information about their data collection on e-Babylab. 


\section{References}

Ackermann, L., Lo, C. H., Mani, N., \& Mayor, J. (2020). Word learning from a tablet app: Toddlers perform better in a passive context. PLoS ONE, 15(12), e0240519. doi: 10.1371/journal.pone.0240519

Anwyl-Irvine, A. L., Massonnié, J., Flitton, A., Kirkham, N., \& Evershed, J. K. (2020). Gorilla in our midst: An online behavioral experiment builder. Behavior research methods, 52(1), 388-407. doi: 10.3758/s13428-019-01237-х

Bates, D., Mächler, M., Bolker, B., \& Walker, S. (2015). Fitting Linear Mixed-Effects Models Using lme4. [sparse matrix methods; linear mixed models; penalized least squares; Cholesky decomposition]. 2015, 67(1), 48. doi: 10.18637/jss.v067.i01

Bobb, S. C., Huettig, F., \& Mani, N. (2016). Predicting visual information during sentence processing: Toddlers activate an object's shape before it is mentioned. [journal article]. Journal of Experimental Child Psychology, 151, 51-64. doi: 10.1016/j.jecp.2015.11.002

Borovsky, A., Elman, J. L., \& Fernald, A. (2012). Knowing a lot for one's age: Vocabulary skill and not age is associated with anticipatory incremental sentence interpretation in children and adults. Journal of Experimental Child Psychology, 112(4), 417-436. doi: 10.1016/j.jecp.2012.01.005

Bothe, R., Trouillet, L., Elsner, B., \& Mani, N. (in prep). The interplay of word and category knowledge on learning.

Chai, J. H., Lo, C. H., \& Mayor, J. (2020). A Bayesian-Inspired Item Response Theory-Based Framework to Produce Very Short Versions of MacArthur-Bates Communicative Development Inventories. Journal of Speech, Language, and Hearing Research, 63(10), 3488-3500. doi: 10.1044/2020_JSLHR-20-00361 
de Leeuw, J. R. (2015). jsPsych: A JavaScript library for creating behavioral experiments in a Web browser. Behavior research methods, 47(1), 1-12. doi: 10.3758/s13428-0140458-y

Frank, M. C., Bergelson, E., Bergmann, C., Cristia, A., Floccia, C., Gervain, J., . . Yurovsky, D. (2017). A Collaborative Approach to Infant Research: Promoting Reproducibility, Best Practices, and Theory-Building. Infancy, 22(4), 421-435. doi: 10.1111/infa.12182

Golinkoff, R. M., Hirsh-Pasek, K., Cauley, K. M., \& Gordon, L. (1987). The eyes have it: lexical and syntactic comprehension in a new paradigm. Journal of Child Language, 14(1), 23-45. doi: 10.1017/s030500090001271x

Golinkoff, R. M., Ma, W., Song, L., \& Hirsh-Pasek, K. (2013). Twenty-Five Years Using the Intermodal Preferential Looking Paradigm to Study Language Acquisition:What Have We Learned? Perspectives on Psychological Science, 8(3), 316-339. doi: $10.1177 / 1745691613484936$

Gosling, S. D., Vazire, S., Srivastava, S., \& John, O. P. (2004). Should we trust web-based studies? A comparative analysis of six preconceptions about internet questionnaires. The American psychologist, 59(2), 93-104. doi: 10.1037/0003-066x.59.2.93

Henrich, J., Heine, S. J., \& Norenzayan, A. (2010). The weirdest people in the world? Behavioral and Brain Sciences, 33(2-3), 61-83. doi: 10.1017/s0140525x0999152x

Hewson, C. M., Laurent, D., \& Vogel, C. M. (1996). Proper methodologies for psychological and sociological studies conducted via the Internet. Behavior Research Methods, Instruments, \& Computers, 28(2), 186-191. doi: 10.3758/bf03204763

Kraut, R., Olson, J., Banaji, M., Bruckman, A., Cohen, J., \& Couper, M. (2004). Psychological research online: report of Board of Scientific Affairs' Advisory Group on the Conduct of Research on the Internet. American psychologist, 59(2), 105-117. doi: 10.1037/0003-066x.59.2.105 
Lausberg, H., \& Sloetjes, H. (2009). Coding gestural behavior with the NEUROGES-ELAN system. Behavior research methods, 41(3), 841-849. doi: 10.3758/brm.41.3.841

Lo, C. H., Rosslund, A., Chai, J. H., Mayor, J., \& Kartushina, N. (in press). Tablet Assessment of Word Comprehension Reveals Coarse Word Representations in 18-20month-old Toddlers. psyarxiv.com. doi: 10.31234/osf.io/7hpgm

Lourenco, S. F., \& Tasimi, A. (2020). No Participant Left Behind: Conducting Science During COVID-19. Trends in Cognitive Sciences, 24(8), 583-584. doi: https://doi.org/10.1016/j.tics.2020.05.003

Mani, N., Daum, M. M., \& Huettig, F. (2016). "Proactive" in many ways: Developmental evidence for a dynamic pluralistic approach to prediction. Quarterly Journal of Experimental Psychology, 69(11), 2189-2201. doi: 10.1080/17470218.2015.1111395

Mani, N., \& Huettig, F. (2012). Prediction During Language Processing is a Piece of Cake But Only for Skilled Producers. [journal article]. Journal of Experimental Psychology. Human Perception \& Performance, 38(4), 843-847. doi: 10.1037/a0029284

Mayor, J., \& Mani, N. (2019). A short version of the MacArthur-Bates Communicative Development Inventories with high validity. Behavior research methods, 51(5), 22482255. doi: 10.3758/s13428-018-1146-0

NetMarketShare. (2021). Market Share Statistics for Internet Technologies, from https://bit.ly/2N0JYIm

Rhodes, M., Rizzo, M. T., Foster-Hanson, E., Moty, K., Leshin, R. A., Wang, M., . . Ocampo, J. D. (2020). Advancing Developmental Science via Unmoderated Remote Research with Children. Journal of Cognition and Development, 1-17. doi: $10.1080 / 15248372.2020 .1797751$

Santolin, C., Garcia-Castro, G., Zettersten, M., Sebastian-Galles, N., \& Saffran, J. R. (2021). Experience with research paradigms relates to infants' direction of preference. Infancy, 26(1), 39-46. doi: https://doi.org/10.1111/infa.12372 
Sauter, M., Draschkow, D., \& Mack, W. (2020). Building, Hosting and Recruiting: A Brief Introduction to Running Behavioral Experiments Online. Brain Sciences, 10(4). doi: 10.3390/brainsci10040251

Scott, K., \& Schulz, L. (2017). Lookit (Part 1): A New Online Platform for Developmental Research. Open Mind, 1, 4-14. doi: 10.1162/OPMI_a_00002

Semmelmann, K., Hönekopp, A., \& Weigelt, S. (2017). Looking Tasks Online: Utilizing Webcams to Collect Video Data from Home. [10.3389/fpsyg.2017.01582]. Frontiers in Psychology, 8, 1582.

Sheskin, M., \& Keil, F. (2018). TheChildLab. com a video chat platform for developmental research. psyarxiv.com. doi: 10.31234/osf.io/rn7w5

Sheskin, M., Scott, K., Mills, C. M., Bergelson, E., Bonawitz, E., Spelke, E. S., . . Schulz, L. (2020). Online Developmental Science to Foster Innovation, Access, and Impact. Trends in Cognitive Sciences, 24(9), 675-678. doi: https://doi.org/10.1016/j.tics.2020.06.004

Simonsen, H. G., Kristoffersen, K. E., Bleses, D., Wehberg, S., \& Jørgensen, R. N. (2014). The Norwegian Communicative Development Inventories: Reliability, main developmental trends and gender differences. First Language, 34(1), 3-23. doi: $10.1177 / 0142723713510997$

Spelke, E. S. (1979). Perceiving bimodally specified events in infancy. Developmental Psychology, 15, 626-636. doi: 10.1037/0012-1649.15.6.626 


\section{Tables}

Table 1. Supported file types and extensions

\begin{tabular}{ll}
\hline File type & File extensions \\
\hline Audio & $. \mathrm{mp} 3,$. wav \\
Document & .css, .csv, .docx, .html, .pdf, .rtf, .tpl, .txt, .xlsx \\
Image &. gif, .jpeg, .jpg, .png \\
Video & $. \mathrm{mp} 4,$. ogg, .webm \\
\hline
\end{tabular}


Table 2. Use of e-Babylab for the studies conducted in BabyLing lab, Oslo (2019-Jan. 2021)

\begin{tabular}{|c|c|c|c|c|c|c|c|c|c|}
\hline Task & Study & Stimuli & Age & $\mathrm{N}$ & $\begin{array}{l}\text { Response } \\
\text { type }\end{array}$ & Trials* & $\begin{array}{l}\text { Assessment } \\
\text { place }\end{array}$ & Device & $\begin{array}{c}\text { Links to OSF/papers and } \\
\text { notes }\end{array}$ \\
\hline $4 \mathrm{AFC}$ & $\begin{array}{l}\text { Emotion } \\
\text { word } \\
\text { recognition }\end{array}$ & $\begin{array}{l}\text { Pictures of faces and } \\
\text { audio prompts }\end{array}$ & $2-5$ years & 200 & Touch & $8-12$ & $\begin{array}{l}\text { Kindergarte } \\
\mathrm{n}\end{array}$ & $\begin{array}{l}\text { Samsung } \\
\text { Galaxy TabA } \\
10.5^{\prime \prime}\end{array}$ & (PsyArxiv to appear) \\
\hline $\begin{array}{l}\text { Namin } \\
\mathrm{g}\end{array}$ & $\begin{array}{l}\text { Emotion } \\
\text { word } \\
\text { production }\end{array}$ & $\begin{array}{l}\text { Pictures of emotion } \\
\text { faces and audio } \\
\text { prompts }\end{array}$ & $2-5$ years & 200 & $\begin{array}{l}\text { Audio } \\
\text { recording }\end{array}$ & $8-12$ & $\begin{array}{l}\text { Kindergarte } \\
\mathrm{n}\end{array}$ & $\begin{array}{l}\text { Samsung } \\
\text { Galaxy Tab A } \\
10.5^{\prime \prime}\end{array}$ & $\begin{array}{l}\text { 2-year olds were shy; } \\
\text { many refused to name } \\
\text { items aloud. }\end{array}$ \\
\hline $4 \mathrm{AFC}$ & $\begin{array}{l}\text { Word } \\
\text { learning via } \\
\text { e-book }\end{array}$ & $\begin{array}{l}\text { Pictures of novel } \\
\text { objects and audio } \\
\text { prompts }\end{array}$ & 2-3 years & $\begin{array}{l}55 \times 3 \\
\text { times }\end{array}$ & Touch & 12 & $\begin{array}{l}\text { Kindergarte } \\
\mathrm{n}\end{array}$ & $\begin{array}{l}\text { Samsung } \\
\text { Galaxy Tab A } \\
10.5^{\prime \prime}\end{array}$ & https://osf.io/9f6cg/ \\
\hline $\begin{array}{l}\text { Namin } \\
\mathrm{g}\end{array}$ & $\begin{array}{l}\text { Word } \\
\text { learning via } \\
\text { e-book }\end{array}$ & $\begin{array}{l}\text { Pictures of novel } \\
\text { objects and audio } \\
\text { prompts }\end{array}$ & 2-3 years & 20 & $\begin{array}{l}\text { Audio } \\
\text { recording }\end{array}$ & $8-12$ & $\begin{array}{l}\text { Kindergarte } \\
\mathrm{n}\end{array}$ & $\begin{array}{l}\text { Samsung } \\
\text { Galaxy Tab A } \\
10.5^{\prime \prime} \\
\end{array}$ & $\begin{array}{l}\text { 2-year olds were reluctant } \\
\text { to name unfamiliar } \\
\text { objects. }\end{array}$ \\
\hline $2 \mathrm{AFC}$ & $\begin{array}{l}\text { Mutual } \\
\text { exclusivity }\end{array}$ & $\begin{array}{l}\text { Pictures of novel and } \\
\text { familiar objects }+ \\
\text { audio }\end{array}$ & $\begin{array}{l}18-20 \\
\text { mo }\end{array}$ & 25 & Touch & 20 & $\mathrm{Lab}$ & $\begin{array}{l}\text { Samsung } \\
\text { Galaxy Tab A } \\
10.5^{\prime \prime}\end{array}$ & Currently being processed \\
\hline $2 \mathrm{AFC}$ & $\begin{array}{l}\text { Toddler- } \\
\text { based CDI }\end{array}$ & $\begin{array}{l}\text { Pictures of familiar } \\
\text { objects + audio }\end{array}$ & $\begin{array}{l}18-20 \\
\text { mo }\end{array}$ & 25 & Touch & 48 & $\mathrm{Lab}$ & $\begin{array}{l}\text { Samsung } \\
\text { Galaxy Tab A } \\
10.5^{\prime \prime}\end{array}$ & $\begin{array}{l}\text { https://osf.io/827fb/ } \\
\text { https://psyarxiv.com/7hpg } \\
\mathrm{m} /\end{array}$ \\
\hline $2 \mathrm{AFC}$ & $\begin{array}{l}\text { Online } \\
\text { Toddler- } \\
\text { based CDI }\end{array}$ & $\begin{array}{l}\text { Pictures of familiar } \\
\text { objects }+ \text { audio }\end{array}$ & $\begin{array}{l}18-36 \\
\text { mo }\end{array}$ & 138 & Touch & 48 & $\begin{array}{l}\text { asynchrono } \\
\text { us remote } \\
\text { data } \\
\text { collection }\end{array}$ & $\begin{array}{l}\text { Tablets, } \\
\text { android } \\
\text { phones, PC }\end{array}$ & $\begin{array}{l}\underline{\mathrm{https}: / / \text { osf.io/827fb/ }} \\
\underline{\mathrm{https} / / \mathrm{psyarxiv.com} / 7 \mathrm{hpg}}\end{array}$ \\
\hline $2 \mathrm{AFC}$ & $\begin{array}{l}\text { Active/passi } \\
\text { ve learning }\end{array}$ & $\begin{array}{l}\text { Pictures of } \\
\text { unfamiliar objects }\end{array}$ & 3.5 years & 50 & Touch & 26 & $\begin{array}{l}\text { Kindergarte } \\
\mathrm{n}\end{array}$ & $\begin{array}{l}\text { Tablet } \\
1280 \times 800\end{array}$ & \\
\hline $4 \mathrm{AFC}$ & $\begin{array}{l}\text { Online } \\
\text { Word } \\
\text { learning }\end{array}$ & $\begin{array}{l}\text { Videos, pictures and } \\
\text { audio prompts }\end{array}$ & 2.5 years & $\begin{array}{l}37 \times 5 \\
\text { times }\end{array}$ & Touch & 12 & $\begin{array}{l}\text { At home } \\
\text { with parents }\end{array}$ & $\begin{array}{l}\text { Tablets, } \\
\text { android } \\
\text { phones, PC }\end{array}$ & $\begin{array}{l}\text { Fully on-line longitudinal } \\
\text { (1-week) study }\end{array}$ \\
\hline
\end{tabular}

Notes: * excluding familiarization trials 
Table 3. LMM results for (log-transformed) reaction times

\begin{tabular}{lccccc}
\hline & $\beta$ & $S E$ & $D F$ & $t$ & $p$ \\
\hline Intercept & 1.955 & 0.075 & 156.439 & 25.794 & $<0.001 * * *$ \\
Age & -0.008 & 0.001 & 101.358 & -6.149 & $<0.001 * * *$ \\
Time 2 & -0.083 & 0.035 & 102.043 & -2.366 & $0.0198 *$ \\
Trial & -0.055 & 0.010 & 469.126 & -5.047 & $<0.001 * * *$ \\
\hline
\end{tabular}

Table 4. Descriptive statistics for response latencies as a function of time and age.

\begin{tabular}{|c|c|c|c|c|}
\hline Age (years) & Time & $\mathrm{N}$ & Mean (s) & $95 \% \mathrm{CI}$ \\
\hline 2 & $\mathrm{~T} 1$ & 16 & 5.26 & $\begin{array}{c}{[4.83,} \\
5.69]\end{array}$ \\
\hline 2 & $\mathrm{~T} 2$ & 11 & 4.58 & $\begin{array}{c}{[3.95,} \\
5.20]\end{array}$ \\
\hline 3 & $\mathrm{~T} 1$ & 21 & 4.53 & $\begin{array}{c}{[3.86,} \\
5.21]\end{array}$ \\
\hline 3 & $\mathrm{~T} 2$ & 20 & 4.35 & $\begin{array}{c}{[3.96,} \\
4.75]\end{array}$ \\
\hline 4 & $\mathrm{~T} 1$ & 21 & 4.28 & $\begin{array}{c}{[3.81,} \\
4.76]\end{array}$ \\
\hline 4 & $\mathrm{~T} 2$ & 22 & 3.76 & $\begin{array}{c}{[3.34,} \\
4.17]\end{array}$ \\
\hline 5 & $\mathrm{~T} 1$ & 18 & 4.07 & $\begin{array}{c}{[3.42,} \\
4.72]\end{array}$ \\
\hline 5 & $\mathrm{~T} 2$ & 20 & 3.54 & $\begin{array}{l}{[3.15,} \\
3.92]\end{array}$ \\
\hline 6 & $\mathrm{~T} 2$ & 8 & 3.53 & $\begin{array}{l}{[2.64,} \\
4.43]\end{array}$ \\
\hline
\end{tabular}


Figures

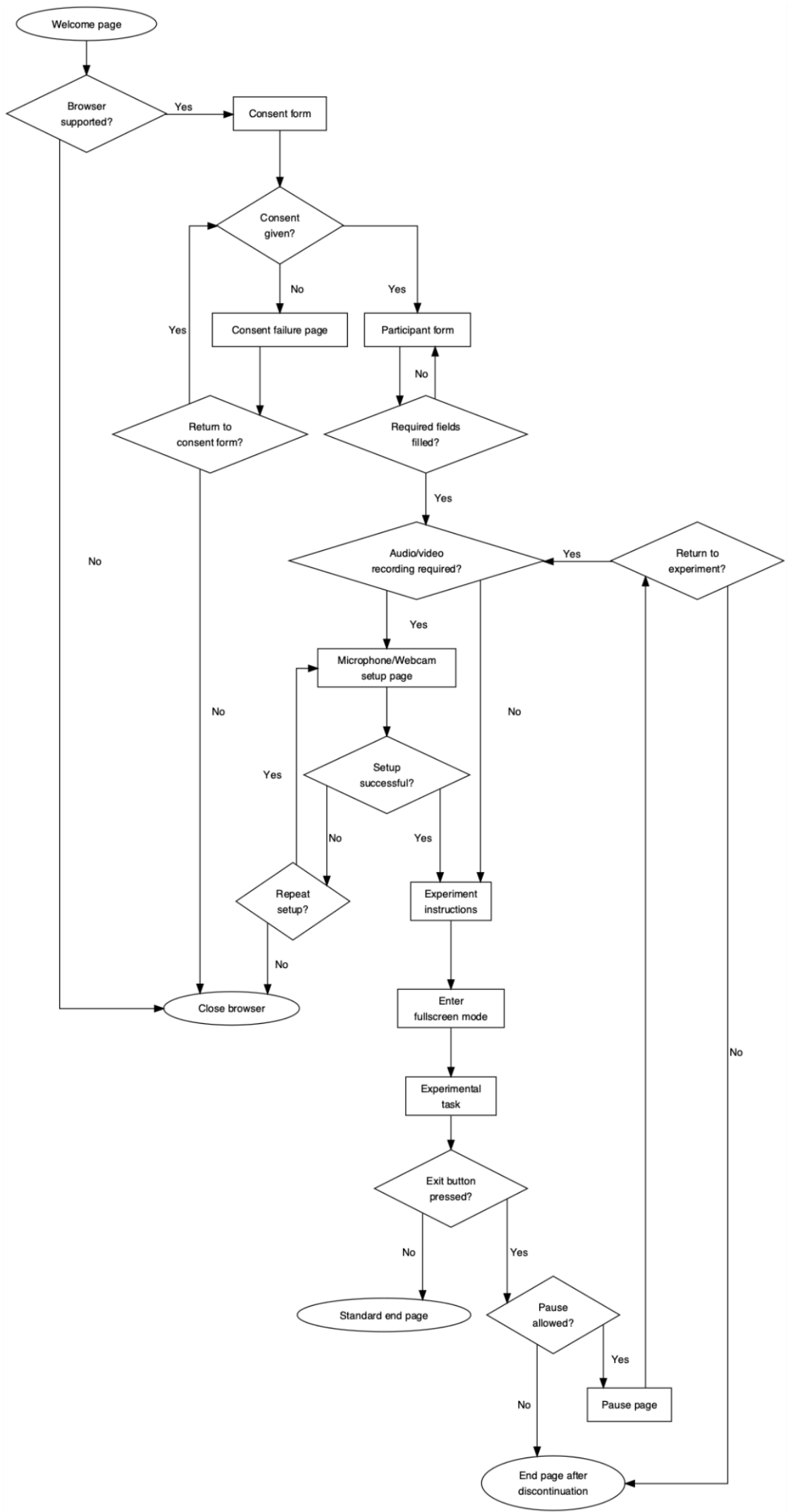

Figure 1. Study procedure. 


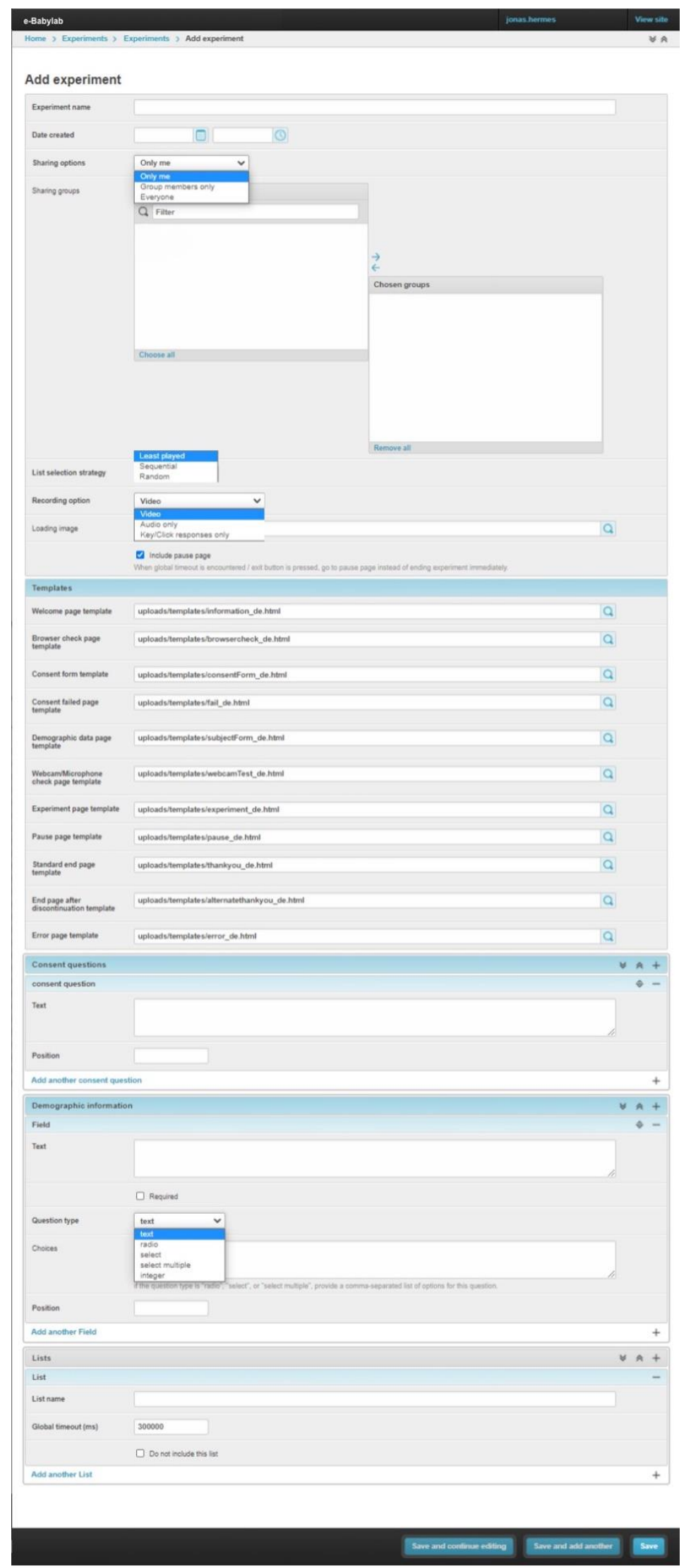

Figure 2. Experiment Wizard. 


\section{Lists}

- Test conditions

- one list played per participant

- List choice options:

random, sequential, least

played.

- lists can be deactivated.

\section{$\Rightarrow$ Outer blocks}

- Played sequentially

- Should start with a video consent item

\section{$\Rightarrow$ Inner blocks}

- Sequential or random order

\section{$\Rightarrow$ Test trials}

- Sequential or random order

- Can contain pictures, video, audio

- Can require responses such as key presses,

Figure 3. Four-level structure of an experimental task.

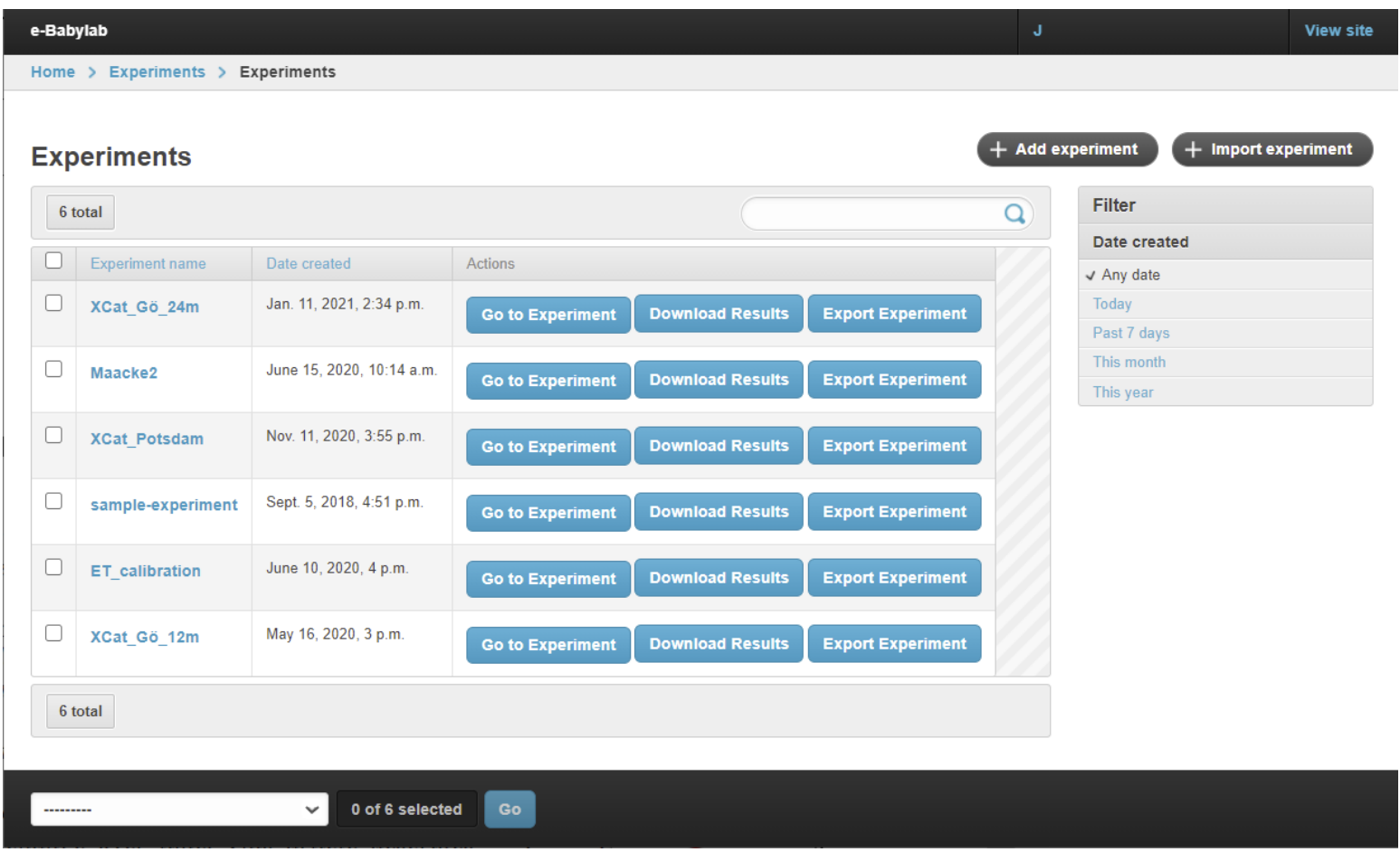

Figure 4. Experiment administration. 
Home > Experiments > Participant data

\section{Participant data}

\begin{tabular}{|c|c|c|c|c|c|c|c|}
\hline 16 results & \multicolumn{5}{|l|}{77 total } & \multirow[t]{2}{*}{ Filter } & \multirow[t]{2}{*}{$\checkmark$} \\
\hline 0 & Participant Number & $2 \wedge$ & Experiment & Listitem & Created & & \\
\hline 0 & 2 & & sample-experiment & List1 & May 23, 2020, 10:07 p.m. & & \\
\hline$\square$ & 3 & & sample-experiment & List1 & May 24, 2020, $12: 24$ p.m. & & \\
\hline 0 & 5 & & sample-experiment & List1 & May $25,2020,12: 41$ p.m. & & \\
\hline 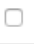 & 6 & & sample-experiment & List1 & May $25,2020,12: 44$ p.m. & & \\
\hline$\square$ & 7 & & sample-experiment & List1 & May 25, 2020, 2:16 p.m. & & \\
\hline$\square$ & 10 & & sample-experiment & - & June 8, 2020, 7:07 p.m. & & \\
\hline 0 & 11 & & sample-experiment & - & June $10,2020,11: 28$ p.m. & & \\
\hline ○ & 12 & & sample-experiment & List1 & June $25,2020,5: 43$ p.m. & & \\
\hline$\square$ & 13 & & sample-experiment & List1 & June 29, 2020, 11:47 a.m. & & \\
\hline ○ & 14 & & sample-experiment & List1 & June 29, 2020, 11:52 a.m. & & \\
\hline
\end{tabular}

Figure 5. Participant data administration.

\begin{tabular}{|l|l|l|l|l}
\hline e-Babylab & Chang Huan
\end{tabular}

Home > FileBrowser

FileBrowser

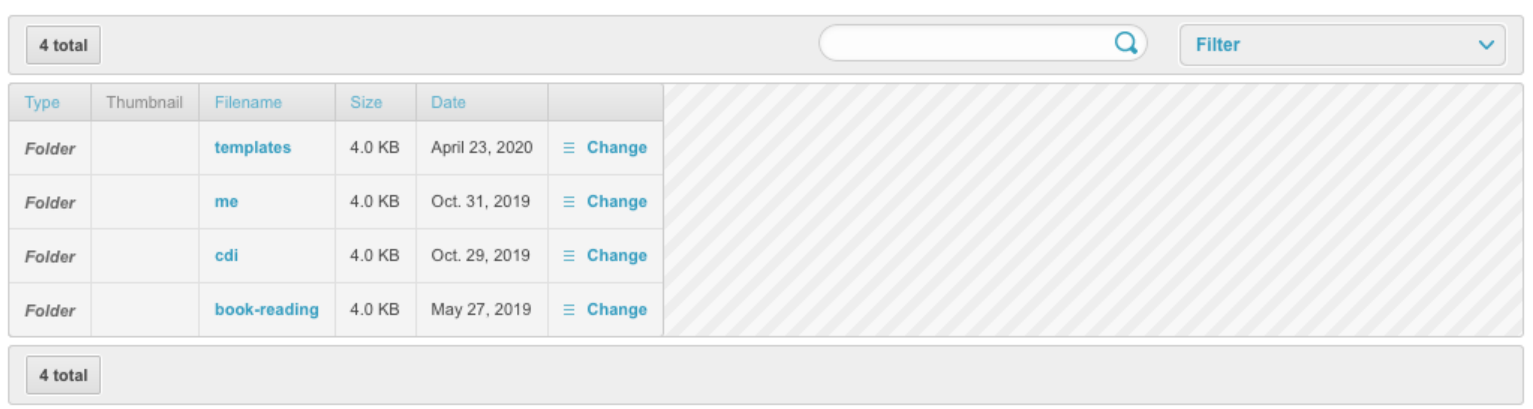

Figure 6. File browser. 

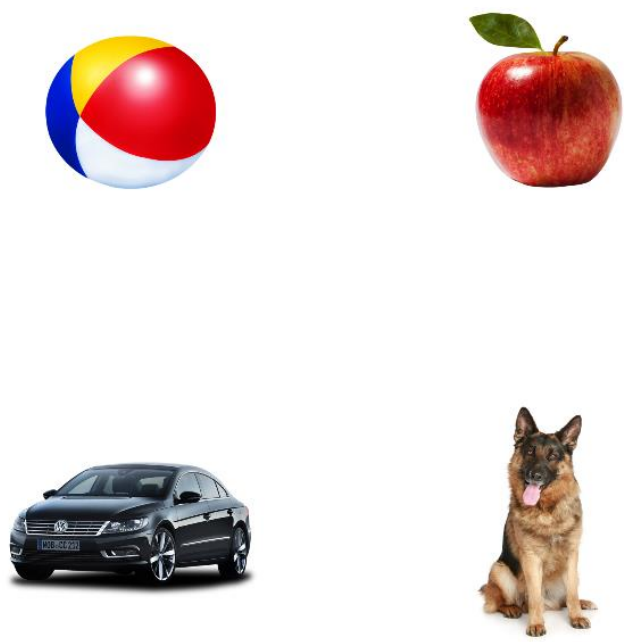

Figure 7. Familiar items used in the 4-forced-choice word recognition task.

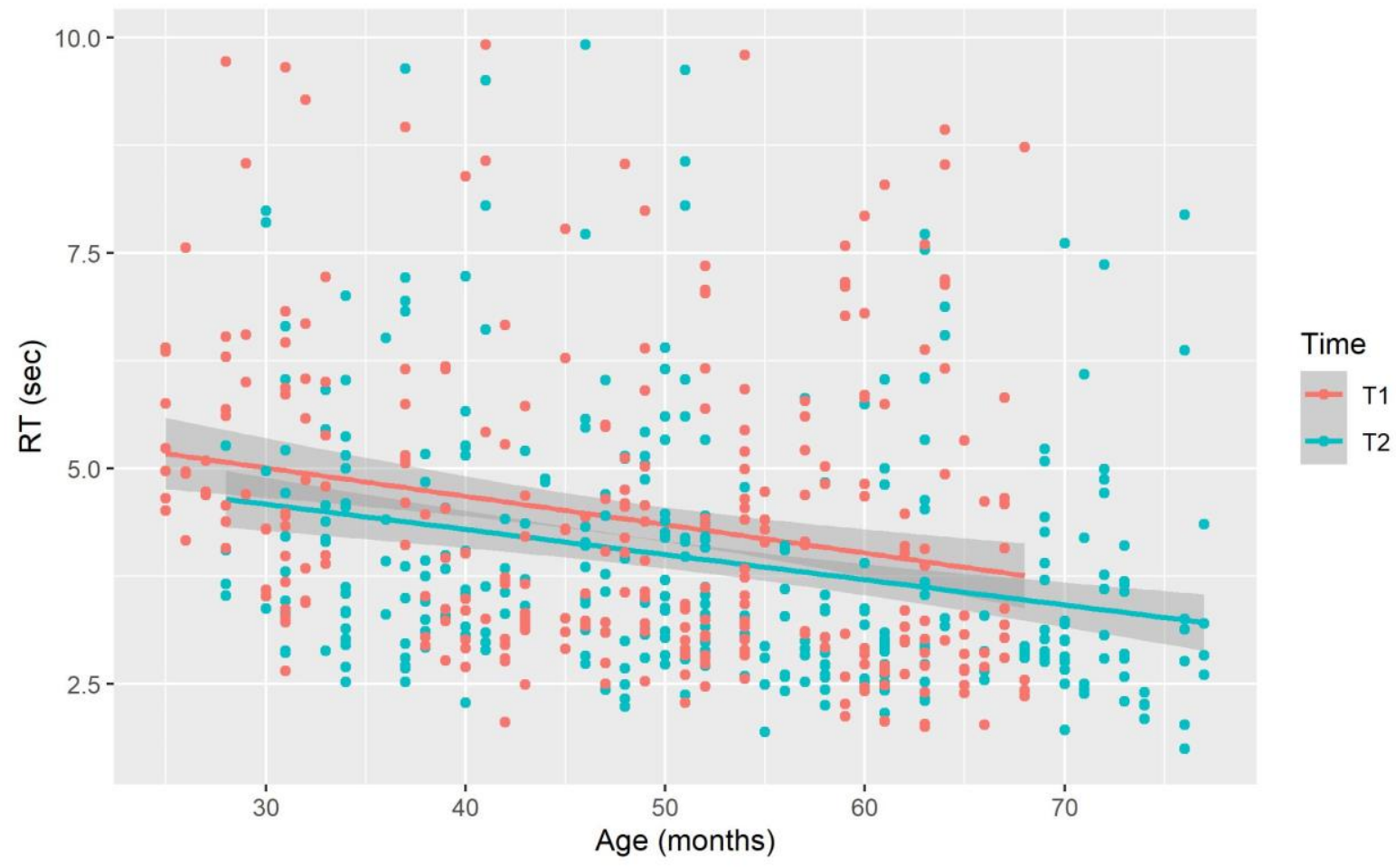

Figure 8. Response latencies at Time 1 and Time 2 across children's ages. 


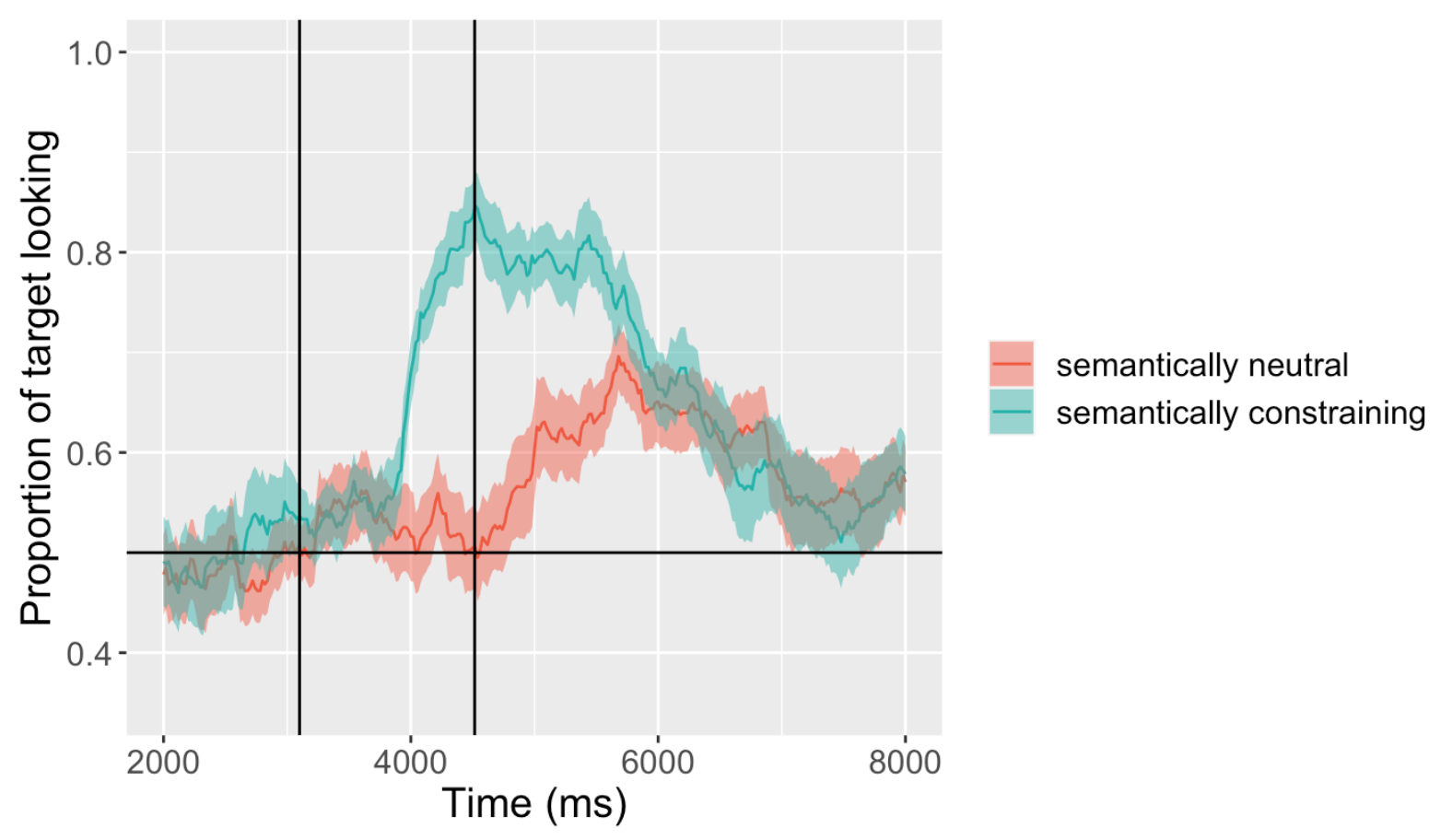

Figure 9. Time-course of fixations to the target across the critical time window in the two conditions. Vertical lines indicate the onset of the verb and the earliest onset of the noun. 


\section{Appendix A. Technical Underpinnings of e-Babylab}

\section{Microservices and Docker}

e-Babylab is developed using a microservices architecture. Contrary to the commonly used monolithic architecture where all the components of an application are developed as a single entity and run in the same process, the microservices architecture centers on developing an application as a set of lightweight and loosely coupled services (or small applications), each running in its own process and serving a specific purpose (see Figure A1; Lewis \& Fowler, 2014). As a result, services of the same application can be developed, deployed, and maintained independently - and rapidly. The independence of services also means that the failure of a single service will not affect other services (i.e., the rest of the application remains functional). Moreover, services can be reused and applied to other applications, thus reducing development costs.

A monolithic application puts all its functionality into a single process...

... and scales by replicating the
monolith on multiple servers
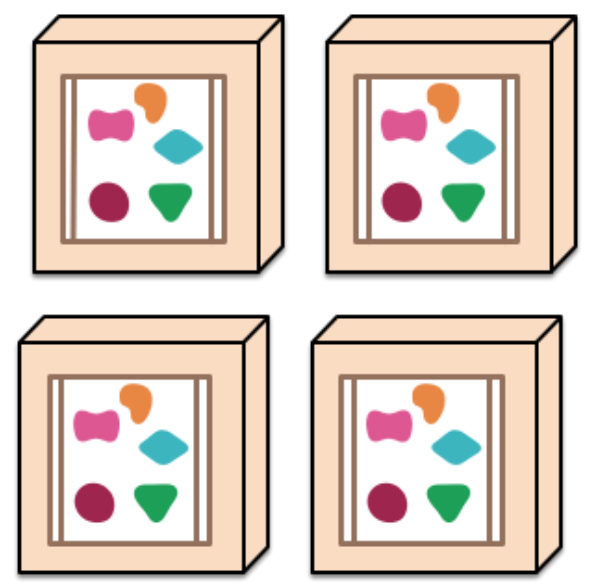

A microservices architecture puts each element of functionality into a separate service...

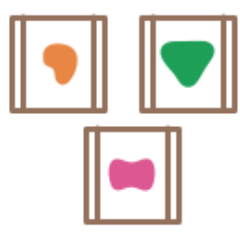

... and scales by distributing these services across servers, replicating as needed.
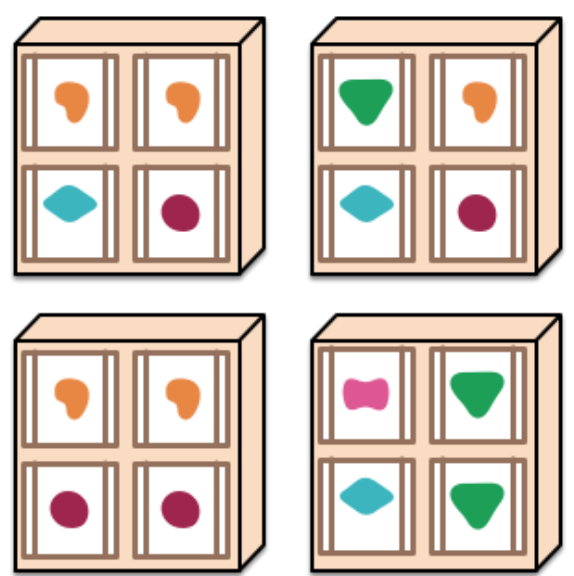

Figure A1. Monoliths and microservices (taken from Lewis \& Fowler, 2014).

Microservices lend themselves well to operating system-level virtualization (also known as containerization), which involves bundling the application code with all its libraries, 
system tools, configuration files, and dependencies (with the exception of the operating system) so that the application will always run the same, regardless of the computing environment (IBM Cloud Education, 2019). Such bundles, referred to as containers, are lightweight in that they share the host machine's operating system kernel, effectively eliminating the overhead of running multiple operating systems. This further translates into faster start-up times and smaller memory footprints. For these reasons, Docker (https://www.docker.com), an open source, lightweight container virtualization platform that runs on Mac, Windows, and Linux is chosen to deploy the e-Babylab services.

As shown in Figure A2, e-Babylab is built of three services - the application programming interface (API) gateway, the content management system, and the database each encapsulated in a container. The arrows represent dependencies between services, which are started in dependency order. In other words, the database is started before the content management system and lastly, the API gateway. As containers are ephemeral, such that they can be stopped, destroyed, rebuilt, and replaced as needed, data generated or used by containers does not persist when the containers are destroyed. Thus, data that needs to be persisted is stored in volumes managed by Docker on the host machine. In doing so, containers can easily be replaced (e.g., in upgrading a service) without any loss of data.

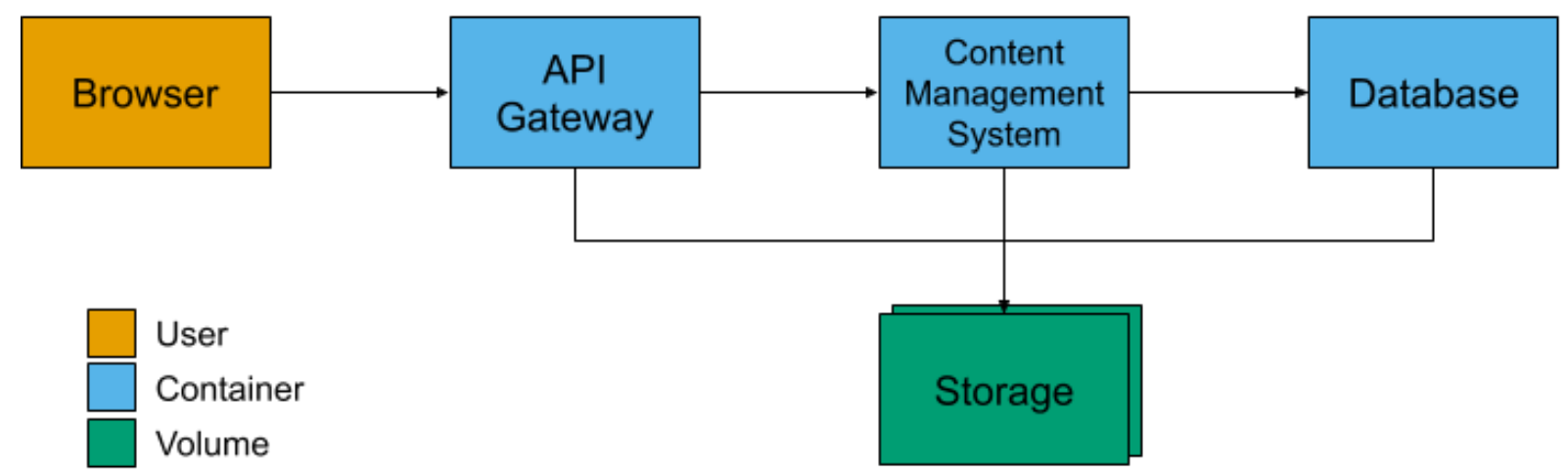

Figure A2. Components of e-Babylab.

Apart from containers, the Docker architecture includes two other major components, namely images and registries. Briefly, containers are created from images which serve as 
blueprints. Each image is defined by a Dockerfile that contains the instructions to create a given image. During a build process, the instructions in a Dockerfile are executed and stored as an image. For ease of distribution and sharing, images can be pushed to registries where images are stored. The Docker-Compose file specifies whether images are to be pulled (i.e., downloaded) from a registry or built locally (using a Dockerfile). The API gateway and the database images of e-Babylab are pulled from Docker Hub (i.e., Docker's public registry) as they can be used as is. On the other hand, as the content management system is heavily customized, the image is built locally.

To orchestrate these services (i.e., to automatically configure, coordinate, and manage them) and start up e-Babylab, Docker Compose is used. By running docker-compose up, Docker Compose pulls the images for the API gateway and the database, builds an image for the content management system, and finally starts and runs the e-Babylab services as defined in the Docker-Compose file.

\section{API Gateway}

The API gateway is implemented using the open source version of NGINX (https://hub.docker.com/_/nginx), a multipurpose web server which also acts as a reverse proxy and Transport Layer Security (TLS) terminator. The API gateway acts as the entry point into e-Babylab and forwards a client's (e.g., browser) requests to the content management system and database services. With the addition of a TLS certificate, this entry point is protected by TLS, the successor to Secure Sockets Layer (SSL), which takes care of securing end-to-end communications (e.g., data transfer) between two systems. Put simply, eBabylab is served over Hypertext Transfer Protocol Secure (HTTPS). Additionally, NGINX is configured to redirect any unsecured Hypertext Transfer Protocol (HTTP) requests to HTTPS.

\section{Content Management System}

\section{Django}


The content management system which provides the administrative interface to manage experiments as well as participant data and results is implemented with Django (https://hub.docker.com/_/django), an open source Python-based web framework. With its aim to encourage rapid development, Django provides a complete set of ready-made components needed in most web development tasks, such as the authentication system and the dynamic administrative interface described earlier. On top of the aforementioned TLS/HTTPS protection, Django provides an extra layer of security by preventing most common security vulnerabilities in web applications, such as cross-site scripting, cross-site request forgery, Structured Query Language (SQL) injection, and clickjacking (see The OWASP Foundation, 2017, for more details on common security vulnerabilities). Thus, focus can be placed on developing the parts of a project that are unique, which in the case of e-Babylab, are the experiments as well as participant data and results.

In order to generate HTML dynamically, both for e-Babylab and the experiment front end, Django's own template system, namely the Django template language is used. Typically, a template contains both static (non-editable) and dynamic (editable) parts of the desired HTML output, allowing the same design to be reused while the content changes dynamically. As shown in Figure A3, Django retrieves data from the database and the file system - where template files, stimuli, and media recordings are stored - and renders (i.e., interpolates) the templates with these data to dynamically display contents on the user-facing administration system and the participant-facing experiment front end. The figure also shows the flow of data in setting up, importing, and exporting experiments; in recording participant data and responses during an experiment; as well as in downloading participant data and results. 


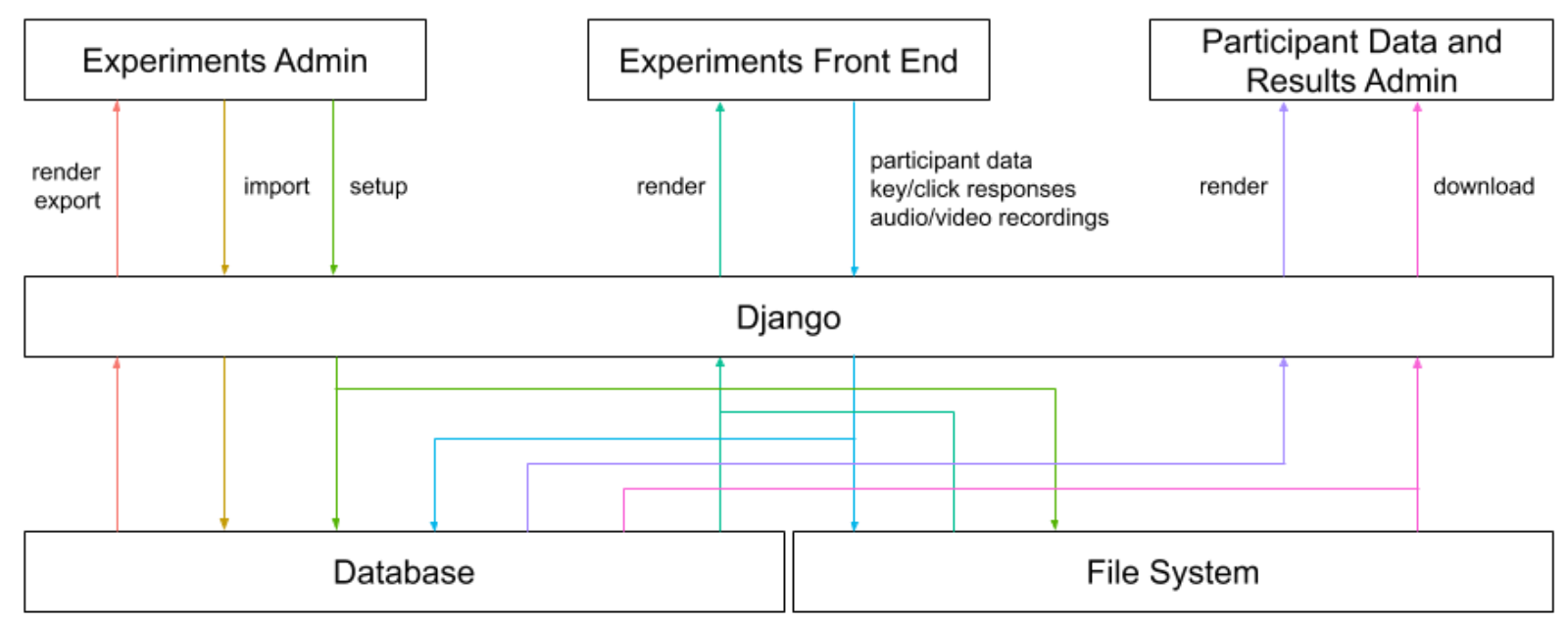

Figure A3. Data flow in the content management system.

\section{Import and Export of an Experiment Setup}

The import and export functions are realized using JavaScript Object Notation (JSON), a lightweight, human-readable, text-only data interchange format used in storing and transporting data. For exporting an experiment setup, all parts of the experiment setup, from the general settings until the trials, are first retrieved from the database and serialized into JSON objects, which are then downloaded as a single JSON file. Likewise, for importing an experiment setup, a user-uploaded JSON file containing JSON objects making up the parts of an experiment setup is simply deserialized and a new experiment is created, set up, and stored in the database.

\section{Media Recording}

An important feature offered in experiments created with e-Babylab is the capability of recording audio and video. This is enabled by the MediaStream Recording API (https://developer.mozilla.org/en-US/docs/Web/API/MediaStream_Recording_API). As the API is only available in Google Chrome and Mozilla Firefox for Android and desktop, experiments programmed with e-Babylab will not run on current iOS devices, such as iPhones and iPads. For this reason, a browser compatibility check is included as part of every experiment. 
Media recording involves both the front end and the back end. On the front end, the getUserMedia() function of the MediaDevices interface asks for permission to use the participant's media input devices (e.g., microphone and/or webcam) and produces a MediaStream object containing audio or video tracks, depending on the type that is requested. This MediaStream object is then passed to a MediaRecorder object which is configured to record media as 1-second chunks to be uploaded via Asynchronous JavaScript and XML (AJAX) to the Django back end. Media is recorded per trial. When the final chunk of a trial is received on the back end, individual chunks are merged as a single media file which is then stored on the file system and referenced in the database. To account for low bandwidth environments, videos are recorded in $640 \times 480$ pixels.

\section{Database}

The database where experiments as well as participant data and results are stored is a relational database created using the open source relational database management system PostgreSQL (https://hub.docker.com/_/postgres). In a relational database, data is stored in tabular form where rows are referred to as records and columns, attributes. Records in different tables can be linked - or related - based on a unique key attribute. With this key, data from multiple tables can be retrieved with a single query. For instance, downloading participant data and results of an experiment requires data to be retrieved from the participant data table, the experiments table, the lists table, the outer-blocks table, and so on. This can be easily achieved using the experiment identifier (ID) which serves as the key. In addition, as PostgreSQL is supported by Django, any changes made to the database schema, such as the addition of new tables, can simply be stored by running python manage.py makemigrations which automatically generates the SQL commands needed to modify the database schema. To execute these commands (i.e., to apply the changes) the python manage.py migrate command is used (see "Django documentation: Migrations", n.d., for more details on Django migrations). 


\section{Appendix B. Sample HTML Template}

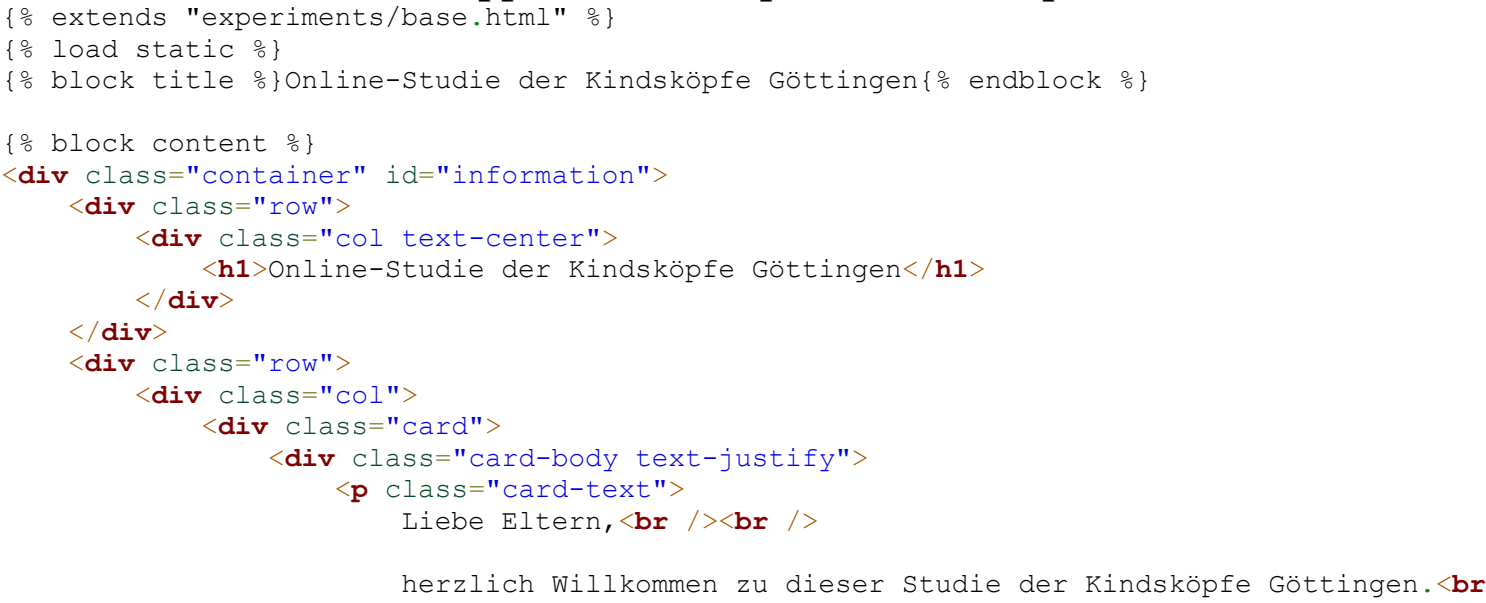

$/><$ br $/>$

Wenn Sie zusammen mit Ihrem Kind teilnehmen möchten, lesen Sie bitte die folgenden Informationen aufmerksam durch: $\langle$ br $/>$

- In dieser Studie untersuchen wir XXX<br $/>$

- Für die Teilnahme muss Ihr Kind XXX alt sein. $<$ br $/>$

- Um diese Online-Studie auswerten zu können, benötigen wir Video-

Mitschnitte. Dafür möchten wir die Webcam Ihres Computers nutzen. Für die Teilnahme müssen Sie a einem Computer oder Laptop mit Webcam sein und dazu bereit sein, diese zur Aufnahme freizugeben. Die Videos werden über eine sichere Verbindung (TLS-Verschlüsselung, 256-Bit)

direkt auf die Server der Universität Göttingen übertragen, wo sie unter höchsten

Sicherheitsstandards gespeichert werden. $\langle$ br $/>$

- Für die Aufnahme muss Ihr Kind auf Ihrem Schoß sitzen und die Webcam muss so ausgerichtet sein, dass Ihr Kind sichtbar ist. $\langle$ br />

- Wir werden Ihnen vorab ein paar Fragen stellen. Sie Speicherung

Ihrer persönlichen Daten erfolgt separat von den Daten und Videos der Studie. $\langle$ br $/>$

- Die Studie ist nur mit den Browsern Firefox und Google Chrome

kompatibel. Bitte nutzen sie einen dieser Browser. $\langle$ br $/>$

- Sie können die Studie jederzeit ohne Begründung abbrechen. Während der gesamten Erhebung wird rechts unten im Bild ein Feld "Abbrechen" sichtbar sein. Klicken Sie dieses an, falls Sie die Studie vorzeitig abbrechen möchten. $\langle$ br $/>$

- Sie können jederzeit die Löschung Ihrer Daten veranlassen. Schreiben Sie dazu eine Email an XXX und nennen Sie den genauen Namen, den Sie ins Namensfeld eingegeben haben. $\langle$ br $/>\langle$ br $/>$

Wenn Sie einverstanden sind, klicken Sie bitte unten auf "Weiter" Bevor es losgeht werden wir Ihnen noch einige Fragen stellen und einige technische Checks durchführen. $\langle$ br $/\rangle\langle$ br $/\rangle$

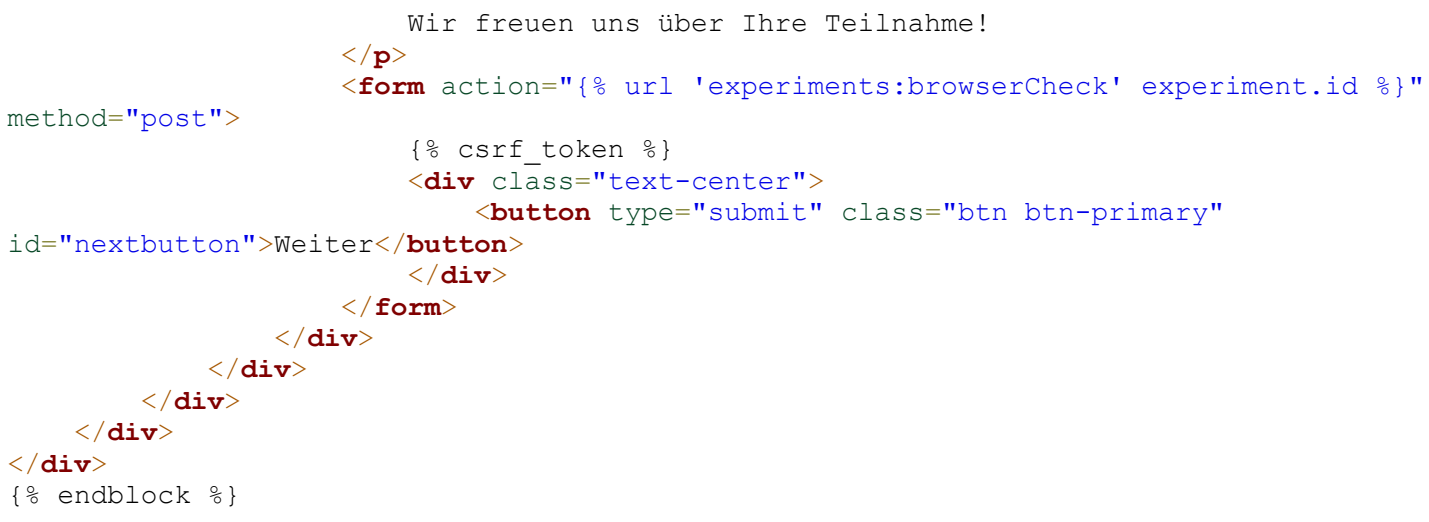

